\title{
Two axiomatic characterizations for the system of spheres-based (and the Epistemic Entrenchment-based) multiple contractions
}

\author{
Maurício D. L. Reis ${ }^{1,2}$ • Pavlos Peppas ${ }^{3,4}$. \\ Eduardo Fermé ${ }^{1,2}$
}

Published online: 7 April 2015

(C) Springer International Publishing Switzerland 2015

\begin{abstract}
In some recent works (Reis 2011, Fermé and Reis, J. Philos. Log. 41, 29-52, 2012, Fermé and Reis, Rev. Symb. Log. 6, 460-487, 2013) two new kinds of multiple contraction functions have been proposed, namely the system of spheres-based multiple contractions and the epistemic entrenchment-based multiple contractions, as generalizations (to the case of multiple contraction) of the well-known classes of systems of spheres-based and of epistemic entrenchment-based (singleton) contractions. Additionally, a representation theorem for the class of epistemic entrenchment-based multiple contraction has been proposed, and it has been shown that the two newly proposed constructions are equivalent, in the sense that a multiple contraction function is a system of spheres-based multiple contraction if and only if it is an epistemic entrenchment-based multiple contraction. In this paper we present two axiomatic characterizations for those multiple contraction functions which differ from the one mentioned above and, in particular, make use of some more intuitive postulates.
\end{abstract}

Maurício D. L. Reis

m_reis@uma.pt

Pavlos Peppas

pavlos.peppas@uts.edu.au

Eduardo Fermé

ferme@uma.pt

1 Universidade da Madeira, Campus Universitário da Penteada, 9020-105 Funchal, Portugal

2 NOVA Laboratory for Computer Science and Informatics (NOVA LINCS), Universidade Nova de Lisboa, Lisboa, Portugal

3 Department of Business Administration, University of Patras, 26500 Patras, Greece

4 QCIS, Faculty of Engineering and IT, University of Technology, Sydney, NSW 2007, Australia 
Keywords Belief change - Theory contraction - Multiple contraction ·

System of spheres · Epistemic entrenchment $\cdot$ Axiomatic characterization

Mathematics Subject Classification (2010) $03 \mathrm{~B} 42 \cdot 68 \mathrm{~T} 27$

\section{Introduction}

One of the central goals of the area known as logic of theory change is to model how information is removed from the epistemic state of an agent. Several proposals have been presented in the literature for modelling operations of that kind - commonly known as contraction functions - which, roughly speaking (and assuming that the beliefs of an agent are represented by sentences), receive a set of beliefs and a sentence that is intended to be removed from it and return a new set of beliefs that no longer contains the given sentence.

In all that follows we assume that the underlying language $\mathcal{L}$ is built from a finite set of propositional symbols and the Boolean connectives $\neg, \wedge, \vee, \rightarrow$ and $\leftrightarrow$. Additionally we shall make use of a consequence operation $C n$ that takes sets of sentences to sets of sentences and which satisfies the standard Tarskian properties, namely inclusion, monotony and iteration, and also the properties of supraclassicality, compactness and deduction. We will use $\mathbf{K}$ to represent a set of sentences that is closed under logical consequence (i.e. $\mathbf{K}=C n(\mathbf{K}))-$ such a set is called a belief set or theory. We shall denote the set of all theories of $\mathcal{L}$ by $\mathcal{T}_{\mathcal{L}}$.

The one which is currently considered the standard model in the belief change literature is known as AGM model - after the initials of its three creators: Carlos Alchourrón, Peter Gärdenfors and David Makinson — and has been originally presented in [1]. In that framework the belief states of an agent are represented by belief sets and a function - from $\mathcal{T}_{\mathcal{L}} \times \mathcal{L}$ to $\mathcal{T}_{\mathcal{L}}$ is a contraction function if and only if, for any belief set $\mathbf{K}$ and any sentences $\alpha$ and $\beta$, the following properties are satisfied:

( $\mathbf{K}-1) \quad \mathbf{K}-\alpha$ is a belief set (i.e. $\mathbf{K}-\alpha=C n(\mathbf{K}-\alpha)$ ).

(Closure)

$(\mathbf{K}-2) \quad \mathbf{K}-\alpha \subseteq \mathbf{K}$.

(Inclusion)

(K-3) If $\alpha \notin \mathbf{K}$, then $\mathbf{K}-\alpha=\mathbf{K}$.

(Vacuity)

(K-4) If $\alpha \notin C n(\emptyset)$, then $\alpha \notin \mathbf{K}-\alpha$.

(Success)

(K-5) If $\alpha \leftrightarrow \beta \in C n(\emptyset)$, then $\mathbf{K}-\alpha=\mathbf{K}-\beta$.

(Extensionality)

$(\mathbf{K}-6) \quad \mathbf{K} \subseteq \operatorname{Cn}((\mathbf{K}-\alpha) \cup\{\alpha\})$.

(Recovery)

The six postulates above are commonly known as basic (AGM) postulates for contraction. Besides from those postulates for contraction, the following two postulates were also presented in [1], referring to the contraction by conjunctions:

$(\mathbf{K}-7) \quad(\mathbf{K}-\alpha) \cap(\mathbf{K}-\beta) \subseteq \mathbf{K}-(\alpha \wedge \beta)$.

(Conjunctive overlap)

$(\mathbf{K}-8) \quad \mathbf{K}-(\alpha \wedge \beta) \subseteq \mathbf{K}-\alpha$ whenever $\alpha \notin \mathbf{K}-(\alpha \wedge \beta)$.

(Conjunctive inclusion)

These two properties are usually called the supplementary (AGM) postulates for contraction.

Almost immediately after the publication of the model proposed by the AGM trio, several constructive models of contraction functions satisfying the basic and the supplementary postulates for contraction have been proposed in the literature. Two of the most wellknown among those models are the system of spheres-based contractions introduced by 
Grove in [8] and the epistemic entrenchment-based contractions proposed by Gärdenfors and Makinson in [6, 7].

Later, several researchers (e.g. $[4,5,9,12])$ have pointed out the need for the consideration of more general contraction operations which, in particular, accounted for the removal of a set of sentences (rather than only a single sentence) from a given belief set. An operation of that kind is now commonly designated by multiple contraction. More precisely, given a belief set $\mathbf{K}$, a multiple contraction on $\mathbf{K}$ is a function that receives any finite set of sentences $B$ and returns (if possible) a new belief set that is contained in $\mathbf{K}$ and which does not contains any of the elements of $B .{ }^{1}$ In what follows, in order to assure that it is always clear which kind of contraction we are referring to at each moment, we will use the expression singleton contraction to designate contractions by a single sentence (like the ones mentioned in the first paragraph above).

One of the strongest arguments provided in the literature supporting the usefulness and the necessity of the study of multiple contractions was the observation, made by Fuhrmann and Hansson in [5], that the sets which are intuitively acceptable as possible results of the multiple contraction of a theory $\mathbf{K}$ by a set of sentences, say $\{\alpha, \beta\}$, are, in general, different from every set which can be the result of either of the following operations:

1. contracting $\mathbf{K}$ by $\alpha \wedge \beta$,

2. contracting $\mathbf{K}$ by $\alpha \vee \beta$,

3. first contracting $\mathbf{K}$ by $\alpha$ and then (contracting the result of such contraction) by $\beta$, or vice versa,

4. intersecting the results of contracting $\mathbf{K}$ by $\alpha$ and of contracting $\mathbf{K}$ by $\beta$.

Furthermore, in that same reference, it has also been stated that, generally speaking, apart from the four above mentioned operations, any other attempt of reducing the multiple contraction of a theory to a certain combination of contractions by a single sentence shall fail.

As a result of the awareness of the need to develop models of theory multiple contractions, several generalizations of the most well-known models of (AGM) singleton contractions were presented in the literature. In particular, the two models of singleton contractions highlighted above have been generalized to the multiple contraction case in $[2,3$, 14], where the classes of system of spheres-based multiple contractions and of epistemic entrenchment-based multiple contractions were introduced. Furthermore, in [3, 14] it was show that those two classes of multiple contraction functions are identical and an axiomatic characterization for the epistemic entrenchment-based multiple contractions was provided. However, and as it was indeed already noticed in [3, 14], one of the postulates included in that axiomatic characterization - namely the postulate of package singleton reduction is rather technical and suffers from some lack of naturalness.

In this paper we will present two axiomatic characterizations for the class of system of spheres-based multiple contractions and, consequently, also for the class of epistemic entrenchment-based multiple contractions, alternative to the one that has been presented in $[3,14]$. In that regard we highlight here that in these new representation theorems for those classes of functions the postulate of package singleton reduction is replaced by other three more intuitively appealing multiple contraction properties.

\footnotetext{
${ }^{1}$ In [5] a (multiple) contraction function of this kind is designated by package contraction.
} 
The paper is organized as follows: In Section 2 we provide the notation and background needed for the rest of the paper. In particular, there we briefly recall the main definitions and results concerning the above mentioned classes of (singleton and multiple) contraction functions. Then, in Section 3 we propose two new axiomatic characterization for the class of system of spheres-based (and epistemic entrenchment-based) multiple contractions. Finally, in Section 4 we briefly summarize and discuss the main contributions of the paper.

\section{Background}

\subsection{Basic notations and conventions}

In this subsection we briefly introduce the notations and conventions that we will use throughout this paper (besides the ones already mentioned in the previous section).

We will sometimes use $C n(\alpha)$ for $C n(\{\alpha\}), A \vdash \alpha$ for $\alpha \in C n(A), \vdash \alpha$ for $\alpha \in C n(\emptyset)$, $A \nvdash \alpha$ for $\alpha \notin C n(A), \forall \alpha$ for $\alpha \notin C n(\emptyset)$. The letters $\alpha, \alpha_{i}, \beta, \ldots$, will be used to denote sentences. $T$ stands for an arbitrary tautology and $\perp$ for an arbitrary contradiction. $A, A_{i}, B, \ldots$ shall denote subsets of sentences of $\mathcal{L}$. We shall use $\mathbf{K}_{\perp}$ to denote the inconsistent belief set (containing all $\mathcal{L}$-sentences). We shall denote the set of all maximal consistent subsets of $\mathcal{L}$ by $\mathcal{M}_{\mathcal{L}}$. We will use the expression possible world (or just world) to designate an element of $\mathcal{M}_{\mathcal{L}}$. Given a possible world $W$, we shall denote by $\bigwedge W$ the conjunction of all literal in $W . \mathcal{M}, \mathcal{N}_{i}, \mathcal{W}, \ldots$, (except for $\mathcal{L}$ and $\left.\mathcal{P}\right)$, shall be used to denote subsets of $\mathcal{M}_{\mathcal{L}}$. Such sets are called propositions. Given a set of sentences $R$, the set consisting of all the possible worlds that contain $R$ is denoted by $\|R\|$. The elements of $\|R\|$ are the $R$-worlds. $\|\varphi\|$ is an abbreviation of $\|\{\varphi\}\|$ and the elements of $\|\varphi\|$ are the $\varphi$-worlds. To any set of possible worlds $\mathcal{V}$ we associate a belief set $T h(\mathcal{V})$ given by $T h(\mathcal{V})=\bigcap \mathcal{V}$ - under the convention that $\bigcap \emptyset=\mathcal{L} . \mathbb{M}, \mathbb{N}_{i}, \mathbb{W}, \ldots$ shall be used to denote subsets of $\mathcal{P}\left(\mathcal{M}_{\mathcal{L}}\right)$.

\subsection{Singleton contraction}

In what follows we recall two of the most well-known models of singleton contraction (i.e. contraction of a belief set $\mathbf{K}$ by a single sentence $\alpha$ ).

\subsubsection{System of spheres-based contractions}

Below we recall the definitions of a system of spheres and of the system of spheres-based contractions presented in [8].

Definition 2.1 ([8]) Let $\mathcal{X}$ be a subset of $\mathcal{M}_{\mathcal{L}}$. A system of spheres (abrev. S.S.), or spheres' system, centred on $\mathcal{X}$ is a collection $\mathbb{S}$ of subsets of $\mathcal{M}_{\mathcal{L}}$, i.e. $\mathbb{S} \subseteq \mathcal{P}\left(\mathcal{M}_{\mathcal{L}}\right)$, that satisfies the following conditions:

(S1) $\mathbb{S}$ is totally ordered with respect to set inclusion; that is, if $\mathcal{U}, \mathcal{V} \in \mathbb{S}$, then $\mathcal{U} \subseteq \mathcal{V}$ or $\mathcal{V} \subseteq \mathcal{U}$.

(S2) $\mathcal{X} \in \mathbb{S}$, and if $\mathcal{U} \in \mathbb{S}$ then $\mathcal{X} \subseteq \mathcal{U}$.

$(\mathbb{S} 3) \quad \mathcal{M}_{\mathcal{L}} \in \mathbb{S}$ (and so it is the largest element of $\mathbb{S}$ ).

(S4) For every $\varphi \in \mathcal{L}$, if there is any element in $\mathbb{S}$ intersecting $\|\varphi\|$ then there is also a smallest element in $\mathbb{S}$ intersecting $\|\varphi\|$. 
The elements of $\mathbb{S}$ are called spheres. For any consistent sentence $\varphi \in \mathcal{L}$, the smallest sphere in $\mathbb{S}$ intersecting $\|\varphi\|$ is denoted by $\mathbb{S}_{\varphi}$ and $f_{\mathbb{S}}(\varphi)$ denotes the set consisting of the $\varphi$-worlds closest to $\mathcal{X}$, i.e.,

$$
f_{\mathbb{S}}(\varphi)=\|\varphi\| \cap \mathbb{S}_{\varphi}
$$

Definition 2.2 ([8]) Let $\mathbf{K}$ be a belief set and $\mathbb{S}$ be a system of spheres centred on $\|\mathbf{K}\|$. The $\mathbb{S}$-based contraction on $\boldsymbol{K}$ is the contraction operation $-_{\mathbb{S}}$ defined, for any $\varphi \in \mathcal{L}$, by:

$$
\mathbf{K}-_{\mathbb{S}} \varphi= \begin{cases}T h\left(\|\mathbf{K}\| \cup f_{\mathbb{S}}(\neg \varphi)\right) & , \text { if } \nvdash \varphi \\ \mathbf{K} & \text {, if } \vdash \varphi .\end{cases}
$$

An operation - on $\mathbf{K}$ is a system of spheres-based contraction on $\boldsymbol{K}$ if and only if there is a system of spheres $\mathbb{S}$ centred on $\|\mathbf{K}\|$, such that, for all sentences $\varphi \in \mathcal{L}, \mathbf{K}-\varphi=\mathbf{K}-\mathbb{S} \varphi$.

To close this subsection we remind that Grove [8] has shown that the class of system of spheres-based contractions is axiomatically characterized by the basic and supplementary AGM postulates for contraction (that we have reproduced in Section 1), as it is stated in the following proposition:

Proposition 2.3 ([8]) Let $\boldsymbol{K}$ be a belief set and - be a contraction function on $\boldsymbol{K}$. Then is a system of spheres-based contraction if and only if it satisfies the postulates of closure, inclusion, vacuity, success, recovery, extensionality, conjunctive overlap, and conjunctive inclusion.

\subsubsection{Epistemic entrenchment-based contractions}

We start by recalling, in the following definition, the concept of epistemic entrenchment relation.

Definition 2.4 ([6, 7]) An ordering of epistemic entrenchment with respect to a belief set $\mathbf{K}$ is a binary relation $\leq$ on $\mathcal{L}$ which satisfies the following postulates:

(EE1) For all $\alpha, \beta, \delta \in \mathcal{L}$, if $\alpha \leq \beta$ and $\beta \leq \delta$ then $\alpha \leq \delta$.

(Transitivity)

(EE2) For all $\alpha, \beta \in \mathcal{L}$, if $\alpha \vdash \beta$ then $\alpha \leq \beta$.

(Dominance)

(EE3) For all $\alpha, \beta \in \mathcal{L}, \alpha \leq \alpha \wedge \beta$ or $\beta \leq \alpha \wedge \beta$.

(EE4) When $\mathbf{K} \neq \mathbf{K}_{\perp}, \alpha \notin \mathbf{K}$ iff $\alpha \leq \beta$ for all $\beta \in \mathcal{L}$.

(Conjunctiveness)

(EE5) If $\beta \leq \alpha$ for all $\beta \in \mathcal{L}$, then $\vdash \alpha$.

(Minimality)

(Maximality)

We shall denote the strict part and the symmetric part of $\leq$ by $<$ and $=_{\leq}$, respectively.

Now we proceed to the presentation of the definition of the epistemic entrenchmentbased contractions which has been introduced in [6, 7].

Definition 2.5 ([6, 7]) Let $\mathbf{K}$ be a belief set and $\leq$ be an epistemic entrenchment relation with respect to $\mathbf{K}$. The $\leq$-based contraction on $\mathbf{K}$ is the contraction operation $-\leq$ defined, for any $\alpha \in \mathcal{L}$, by:

$$
\mathbf{K}-\leq \alpha= \begin{cases}\{\beta \in \mathbf{K}: \alpha<\alpha \vee \beta\} & \text {, if } \forall \alpha \\ \mathbf{K} & \text {, if } \vdash \alpha .\end{cases}
$$


An operation - on $\mathbf{K}$ is an epistemic entrenchment-based contraction on $\mathbf{K}$ if and only if there is an epistemic entrenchment relation with respect to $\mathbf{K}$ such that, for all sentences $\alpha \in \mathcal{L}, \mathbf{K}-\alpha=\mathbf{K}-\leq \alpha$.

The next proposition states that the epistemic entrenchment-based contractions are axiomatically characterized by the basic and supplementary AGM postulates for contraction, whose formulations we have recalled in Section 1.

Proposition 2.6 ([7]) Let $\boldsymbol{K}$ be a belief set and - be a contraction function on $\boldsymbol{K}$. Then - is an epistemic entrenchment-based contraction if and only if it satisfies the (contraction) postulates of closure, inclusion, vacuity, success, recovery, extensionality, conjunctive overlap, and conjunctive inclusion.

\subsubsection{Interrelation Between Systems of Spheres and Epistemic Entrenchment Relations}

It follows immediately from Propositions 2.3 and 2.6 that the class of system of spheresbased contractions coincides with the class of epistemic entrenchment-based contractions. Thus, it is not surprising that there is a very tight interconnection between the two constructions on which those definitions of contraction functions are based, namely the systems of spheres and the epistemic entrenchment relations. In what follows we recall some of the main results so far presented in the literature concerning the interrelation between those two structures.

We start by presenting, in the following proposition from [10], a way of defining an epistemic entrenchment relation by means of a system of spheres:

Proposition 2.7 ([10, page 300]) Let $\boldsymbol{K}$ be a belief set and $\mathbb{S}$ be a system of spheres centred on $\|\boldsymbol{K}\|$. If $\leq$ is the binary relation on $\mathcal{L}$ defined in the following way:

$$
\forall \alpha, \beta \in \mathcal{L}, \alpha \leq \beta \text { iff either } \mathbb{S}_{\neg \alpha} \subseteq \mathbb{S}_{\neg \beta} \text { or } \vdash \beta, \quad(\leq-\mathbb{S})
$$

then $\leq$ is an epistemic entrenchment relation with respect to $\boldsymbol{K}$, i.e., $\leq$ satisfies conditions (EE1)-(EE5).

In the two following propositions it is exposed that condition $(\leq-\mathbb{S})$ is a necessary and sufficient condition for the $\mathbb{S}$-based contraction to coincide with the $\leq$-based contraction, and another condition relating a system of spheres and an epistemic entrenchment relation which is equivalent to that one is introduced.

Proposition 2.8 ([10, 13, 15]) Let $\boldsymbol{K}$ be a belief set, $\mathbb{S}$ be a system of spheres centred on $\|\boldsymbol{K}\|$ and $\leq$ be an epistemic entrenchment relation with respect to $\boldsymbol{K}$. Then the $\leq$-based contraction on $\boldsymbol{K},-_{\leq}(c f$. Definition 2.5$)$, and the $\mathbb{S}$-based contraction on $\boldsymbol{K},-\mathbb{S}(c f$. Definition 2.2), coincide, i.e.,

$$
\forall \varphi \in \mathcal{L}, \boldsymbol{K}-\leq \varphi=\boldsymbol{K}-\mathbb{S} \varphi,
$$

if and only if condition $(\leq-\mathbb{S})$ is satisfied.

Proposition $2.9([14,15])$ Let $\boldsymbol{K}$ be a belief set, $\mathbb{S}$ be a system of spheres centred on $\|\boldsymbol{K}\|$ and $\leq$ be an epistemic entrenchment relation with respect to $\boldsymbol{K}$. Then condition $(\leq-\mathbb{S})$ is satisfied if and only if

$$
\forall \alpha, \beta \in \mathcal{L} \backslash \operatorname{Cn}(\emptyset), \alpha \leq \beta \text { iff } \mathbb{S}_{\neg \alpha} \subseteq \mathbb{S}_{\neg \beta} .
$$


Finally, the following proposition reveals how, given an epistemic entrenchment relation $\leq$, a system of spheres $\mathbb{S}$ can be defined from it, which is such that condition $(\leq-\mathbb{S})$ is satisfied.

Proposition 2.10 ([15]) Let $\boldsymbol{K}$ be a belief set, $\leq$ be an epistemic entrenchment relation with respect to $\boldsymbol{K}$, and $\mathbb{S}^{\prime}$ be the class of subsets of $\mathcal{M}_{\mathcal{L}}$ defined by:

$$
\mathbb{S}^{\prime}=\left\{\mathcal{W}_{\alpha_{i}}: \alpha_{i} \in \mathcal{L}\right\}
$$

where, for any $\alpha_{i} \in \mathcal{L}, \mathcal{W}_{\alpha_{i}}$ is the set defined as follows:

$$
\mathcal{W}_{\alpha_{i}}=\left\|\left\{\alpha \in \mathcal{L}: \alpha_{i}<\alpha\right\}\right\| .
$$

Furthermore, let $\mathbb{S}$ be the class of subsets of $\mathcal{M}_{\mathcal{L}}$ defined by:

$$
\mathbb{S}= \begin{cases}\mathbb{S}^{\prime} & , \text { if } \boldsymbol{K} \neq \boldsymbol{K}_{\perp} \\ \{\emptyset\} \cup \mathbb{S}^{\prime}, & \text { if } \boldsymbol{K}=\boldsymbol{K}_{\perp}\end{cases}
$$

Then the following statements hold:

(i) $\mathbb{S}$ is a system of spheres centred on $\|\boldsymbol{K}\|$.

(ii) $\mathbb{S}$ and $\leq$ satisfy condition $(\leq-\mathbb{S})$.

\subsection{Multiple Contraction}

Below we recall two models of multiple contractions (i.e. contractions of a belief set $\mathbf{K}$ by a finite set of sentences $B$ ) which result of generalizing the models presented in the previous subsection.

\subsubsection{System of spheres-based multiple contractions}

In what follows we present the system of spheres-based multiple contractions which were introduced in $[2,14]$ as a generalization to the case of multiple contraction of Grove's system of spheres-based (singleton) contractions.

We start by recalling the concept of $\mathbb{S}$-based filtration of a set of sentences.

Definition 2.11 ([2, 14]) Let $\mathbf{K}$ be a belief set and $\mathbb{S}$ be a system of spheres centred on $\|\mathbf{K}\|$.

Consider a set of sentences $B=\left\{\alpha_{1}, \alpha_{2}, \ldots, \alpha_{n}\right\} \subseteq \mathcal{L}$ such that $B \backslash C n(\emptyset) \neq \emptyset$.

Denote by $C_{1}, \ldots, C_{m}$ the (different) equivalence classes in the quotient set of ( $B \backslash$ $C n(\emptyset))$ by $\backsim$, i.e. $\left\{C_{1}, \ldots, C_{m}\right\}=(B \backslash C n(\emptyset)) / \backsim$, where $\backsim$ is the equivalence relation on $B \backslash C n(\emptyset)$ defined by:

$$
\forall \alpha, \beta \in B \backslash C n(\emptyset), \alpha \backsim \beta \text { iff } \mathbb{S}_{\neg \alpha}=\mathbb{S}_{\neg \beta},
$$

Moreover, assume that the equivalence classes $C_{1}, \ldots, C_{m}$ are ordered according to the following condition:

$$
\text { If } 1 \leq i<j \leq m \text { then } \forall \alpha_{r} \in C_{i} \forall \alpha_{s} \in C_{j} \mathbb{S}_{\neg \alpha_{s}} \subset \mathbb{S}_{\neg \alpha_{r}} .
$$

Now consider the following list of subsets of $B$ :

$$
\begin{aligned}
& C_{1}^{\prime}=C_{1} \\
& C_{1}^{\prime \prime}=\left\{\alpha_{i} \in C_{1}^{\prime}: \forall \alpha_{j} \in C_{1}^{\prime} f_{\mathbb{S}}\left(\neg \alpha_{j}\right) \not \subset f_{\mathbb{S}}\left(\neg \alpha_{i}\right)\right\} \\
& B_{1}=C_{1}^{\prime \prime}
\end{aligned}
$$


Moreover, if $m>1$ for all $l \in\{2, \ldots, m\}$, let $C_{l}^{\prime}, C_{l}^{\prime \prime}$ and $B_{l}$ be the sets defined by:

$$
\begin{aligned}
C_{l}^{\prime} & =\left\{\alpha_{i} \in C_{l}: \forall \alpha_{j} \in B_{l-1} f_{\mathbb{S}}\left(\neg \alpha_{j}\right) \not \subset\left\|\neg \alpha_{i}\right\|\right\} ; \\
C_{l}^{\prime \prime} & =\left\{\alpha_{i} \in C_{l}^{\prime}: \forall \alpha_{j} \in C_{l}^{\prime} f_{\mathbb{S}}\left(\neg \alpha_{j}\right) \not \subset f_{\mathbb{S}}\left(\neg \alpha_{i}\right)\right\} ; \\
B_{l} & =B_{l-1} \cup C_{l}^{\prime \prime} .
\end{aligned}
$$

The set $B_{\mathbb{S}}=B_{m}$ is the $\mathbb{S}$-based filtration of $B$.

If $D$ is a set of sentences such that $D \subseteq C n(\varnothing)$, then the $\mathbb{S}$-based filtration of $D$ is the empty set and is denoted by $D_{\mathbb{S}}$, i.e. $D_{\mathbb{S}}=\emptyset$.

We are now in a position to reproduce the definition of system of spheres-based multiple contraction proposed in $[2,14]$.

Definition $2.12([2,14])$ Let $\mathbf{K}$ be a belief set and $\mathbb{S}$ be a system of spheres centred on $\|\mathbf{K}\|$. The $\mathbb{S}$-based multiple contraction on $\boldsymbol{K}$ is the multiple contraction function $\div \mathbb{S}$ defined by:

$$
\mathbf{K} \div \mathbb{S} B= \begin{cases}\operatorname{Th}\left(\|\mathbf{K}\| \cup\left(\cup_{\alpha_{i} \in B_{\mathbb{S}}} f_{\mathbb{S}}\left(\neg \alpha_{i}\right)\right)\right) & \text {, if } B \cap C n(\emptyset)=\emptyset \\ \mathbf{K} & \text {, if } B \cap C n(\emptyset) \neq \emptyset\end{cases}
$$

for any set of sentences $B$ and where $B_{\mathbb{S}}$ is the $\mathbb{S}$-based filtration of $B$.

An operator $\div$ on $\mathbf{K}$ is a system of spheres-based multiple contraction on $\boldsymbol{K}$ if and only if there is a system of spheres $\mathbb{S}$ centred on $\|\mathbf{K}\|$, such that $\mathbf{K} \div B=\mathbf{K} \div \mathbb{S} B$, for any set of sentences $B$.

Next, in order to clarify the definition of system of spheres-based multiple contraction, we present an example.

Example 2.13 Let $\mathcal{L}$ be the propositional language that is built from the three propositional symbols $p, q$ and $r$. Define the worlds $W_{0}, \ldots, W_{7}$ as follows:

$$
\begin{array}{ll}
W_{0}=C n(p \wedge q \wedge r) & W_{4}=C n(\neg p \wedge q \wedge r) \\
W_{1}=C n(p \wedge q \wedge \neg r) & W_{5}=C n(\neg p \wedge q \wedge \neg r) \\
W_{2}=C n(p \wedge \neg q \wedge r) & W_{6}=C n(\neg p \wedge \neg q \wedge r) \\
W_{3}=C n(p \wedge \neg q \wedge \neg r) & W_{7}=C n(\neg p \wedge \neg q \wedge \neg r)
\end{array}
$$

Set $\mathbf{K}=W_{0}$ and consider the sentences $\alpha=\neg\left(\bigwedge W_{3}\right), \beta=\neg\left(\bigwedge W_{1} \vee \wedge W_{2}\right), \delta=$ $\neg\left(\bigwedge W_{2} \vee \wedge W_{3}\right)$ and $\epsilon=\neg\left(\bigwedge W_{3} \vee \wedge W_{4}\right)$. Furthermore, let $\mathbb{S}$ be the following system of spheres centred on $\|\mathbf{K}\|$ :

$$
\begin{aligned}
\mathbb{S}= & \left\{\left\{W_{0}\right\},\left\{W_{0}, W_{1}\right\},\left\{W_{0}, W_{1}, W_{2}\right\},\left\{W_{0}, W_{1}, W_{2}, W_{3}, W_{4}\right\},\right. \\
& \left.\left\{W_{0}, W_{1}, W_{2}, W_{3}, W_{4}, W_{5}, W_{6}, W_{7}\right\}\right\}
\end{aligned}
$$

Figure 1 contains a possible graphical representation of the system of spheres $\mathbb{S}$, where the sets $\|\neg \alpha\|=\left\{W_{3}\right\},\|\neg \beta\|=\left\{W_{1}, W_{2}\right\},\|\neg \delta\|=\left\{W_{2}, W_{3}\right\}$ and $\|\neg \epsilon\|=\left\{W_{3}, W_{4}\right\}$ are highlighted.

Let $\div \mathbb{S}$ be the $\mathbb{S}$-based multiple contraction, and consider the sets $A=\{\alpha, \beta\}$ and $B=$ $\{\beta, \delta, \epsilon\}$. In what follows we illustrate how to obtain the sets $\mathbf{K} \div \mathbb{S} A$ and $\mathbf{K} \div \mathbb{S} B$.

We start by obtaining the former. First of all we need to identify the set $A_{\mathbb{S}}$, i.e. the S-based filtration of $A$.

According to the process described in Definition 2.11 and based on the information exposed in Fig. 1 we obtain that: 


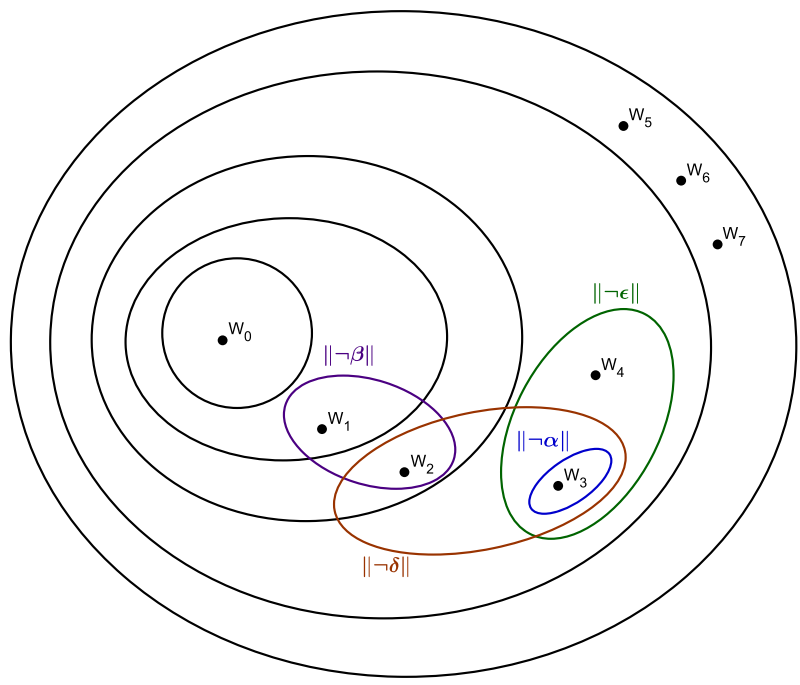

Fig. 1 Schematic representation of the system of spheres $\mathbb{S}$ and the sets $\|\neg \alpha\|,\|\neg \beta\|,\|\neg \delta\|$ and $\|\neg \epsilon\|$ described in Example 2.13

- Since $\mathbb{S}_{\neg \beta} \subset \mathbb{S}_{\neg \alpha}$, the set $(A \backslash C n(\emptyset)) / \sim$ is composed of the following two equivalence classes: $C_{1}=\{\alpha\}$ and $C_{2}=\{\beta\}$ (and, therefore, $m=2$ ).

- $A_{1}=C_{1}^{\prime \prime}=C_{1}^{\prime}=C_{1}=\{\alpha\}$

- $\quad C_{2}^{\prime}=C_{2}=\{\beta\}$ (notice that $f_{\mathbb{S}}(\neg \alpha) \not \subset\|\neg \beta\|$ ).

$-\quad C_{2}^{\prime \prime}=C_{2}^{\prime}=\{\beta\}$.

- $A_{\mathbb{S}}=A_{2}=A_{1} \cup C_{2}^{\prime \prime}=\{\alpha, \beta\}$.

Thus, according to Definition 2.12, we have that $\mathbf{K} \div \mathbb{S} A=$ $T h\left(\|\mathbf{K}\| \cup\left(\bigcup_{\alpha_{i} \in A_{\mathbb{S}}} f_{\mathbb{S}}\left(\neg \alpha_{i}\right)\right)\right)=T h\left(\|\mathbf{K}\| \cup f_{\mathbb{S}}(\neg \alpha) \cup f_{\mathbb{S}}(\neg \beta)\right)=T h\left(\left\{W_{0}, W_{1}, W_{3}\right\}\right)$.

Next we find the $\mathbb{S}$-based filtration $B_{\mathbb{S}}$ of the set $B$ and we obtain the set $\mathbf{K} \div \mathbb{S} B$. So, proceeding as above, we obtain that:

- Since $\mathbb{S}_{\neg \beta} \subset \mathbb{S}_{\neg \delta} \subset \mathbb{S}_{\neg \epsilon}$, the set $(B \backslash C n(\emptyset)) / \sim$ is composed of the following three equivalence classes: $C_{1}=\{\epsilon\}, C_{2}=\{\delta\}$ and $C_{3}=\{\beta\}$ (and, therefore, $m=3$ ).

- $\quad B_{1}=C_{1}^{\prime \prime}=C_{1}^{\prime}=C_{1}=\{\epsilon\}$

- $C_{2}^{\prime}=C_{2}=\{\delta\}$ (notice that $f_{\mathbb{S}}(\neg \epsilon) \not \subset\|\neg \delta\|$ ).

- $C_{2}^{\prime \prime}=C_{2}^{\prime}=\{\delta\}$.

- $\quad B_{2}=B_{1} \cup C_{2}^{\prime \prime}=\{\epsilon, \delta\}$.

- $C_{3}^{\prime}=C_{3} \backslash\{\beta\}=\emptyset$ (notice that $\beta \notin C_{3}^{\prime}$ because $f_{\mathbb{S}}(\neg \delta) \subset\|\neg \beta\|$ ).

- $\quad C_{3}^{\prime \prime}=C_{3}^{\prime}=\emptyset$.

- $B_{\mathbb{S}}=B_{3}=B_{2} \cup C_{3}^{\prime \prime}=\{\epsilon, \delta\}$.

Hence, according to Definition 2.12, we have that $\mathbf{K} \div \mathbb{S} B=$ $\operatorname{Th}\left(\|\mathbf{K}\| \cup\left(\bigcup_{\alpha_{i} \in B_{\mathbb{S}}} f_{\mathbb{S}}\left(\neg \alpha_{i}\right)\right)\right) \quad=\quad \operatorname{Th}\left(\|\mathbf{K}\| \cup f_{\mathbb{S}}(\neg \epsilon) \cup f_{\mathbb{S}}(\neg \delta)\right)=$ $\operatorname{Th}\left(\left\{W_{0}, W_{2}, W_{3}, W_{4}\right\}\right)$.

Finally, the following proposition states that the system of spheres-based multiple contractions are indeed a generalization of Grove's system of spheres-based (singleton) contractions. 
Proposition $2.14([2,14])$ Let $\boldsymbol{K}$ be a belief set and $\mathbb{S}$ be a system of spheres centred on $\|\boldsymbol{K}\|$. If $\div \mathbb{S}$ is the $\mathbb{S}$-based multiple contraction and $-\mathbb{S}$ is the $\mathbb{S}$-based (singleton) contraction, then:

- For any set of sentences $B$ such that $B \cap C n(\emptyset)=\emptyset$ it holds that:

$$
\boldsymbol{K} \div \mathbb{S} B=\bigcap_{\alpha_{i} \in B_{\mathbb{S}}} \boldsymbol{K} \div \mathbb{S}\left\{\alpha_{i}\right\}=\bigcap_{\alpha_{i} \in B_{\mathbb{S}}} \boldsymbol{K}-\mathbb{S} \alpha_{i},
$$

where $B_{\mathbb{S}}$ is the $\mathbb{S}$-based filtration of $B$.

- The identity $\boldsymbol{K} \div \mathbb{S}\{\alpha\}=\boldsymbol{K}-\mathbb{S} \alpha$ is satisfied for any sentence $\alpha \in \mathcal{L}$.

\subsubsection{Epistemic entrenchment-based multiple contractions}

Next we present the definition of epistemic entrenchment-based multiple contractions which was introduced in [3, 14].

First we need to remind the following definition of $\leq$-based filtration of a set of sentences:

Definition 2.15 ([3,14]) Let $\mathbf{K}$ be a belief set and $\leq$ be an epistemic entrenchment relation with respect to $\mathbf{K}$.

Consider a finite set of sentences $B=\left\{\alpha_{1}, \ldots, \alpha_{n}\right\}$ such that $B \backslash C n(\emptyset) \neq \emptyset$.

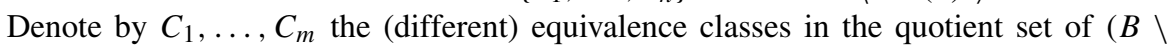
$C n(\emptyset))$ by $\backsim$, i.e. $\left\{C_{1}, \ldots, C_{m}\right\}=(B \backslash C n(\emptyset)) / \backsim$, where $\backsim$ is the equivalence relation on $B \backslash C n(\emptyset)$ defined by:

$$
\forall \alpha, \beta \in B \backslash C n(\emptyset), \alpha \backsim \beta \text { iff } \alpha=\leq \beta,
$$

Moreover, assume that the equivalence classes $C_{1}, \ldots, C_{m}$ are ordered according to the following condition:

$$
\text { If } 1 \leq i<j \leq m \text { then } \forall \alpha_{r} \in C_{i} \forall \alpha_{s} \in C_{j} \alpha_{s}<\alpha_{r} .
$$

Now consider the following list of subsets of $B$ :

$$
\begin{aligned}
& C_{1}^{\prime}=C_{1} \\
& C_{1}^{\prime \prime}=\left\{\alpha_{i} \in C_{1}^{\prime}: \forall \alpha_{j} \in C_{1}^{\prime} \alpha_{j} \vee \neg \alpha_{i} \leq \alpha_{j} \text { or } \alpha_{i}<\alpha_{i} \vee \neg \alpha_{j}\right\} \\
& B_{1}=C_{1}^{\prime \prime}
\end{aligned}
$$

Moreover, if $m>1$ for all $l \in\{2, \ldots, m\}$, let $C_{l}^{\prime}, C_{l}^{\prime \prime}$ and $B_{l}$ be the sets defined by:

$$
\begin{aligned}
C_{l}^{\prime} & =\left\{\alpha_{i} \in C_{l}: \forall \alpha_{j} \in B_{l-1} \alpha_{j} \vee \neg \alpha_{i} \leq \alpha_{j}\right\} ; \\
C_{l}^{\prime \prime} & =\left\{\alpha_{i} \in C_{l}^{\prime}: \forall \alpha_{j} \in C_{l}^{\prime} \alpha_{j} \vee \neg \alpha_{i} \leq \alpha_{j} \text { or } \alpha_{i}<\alpha_{i} \vee \neg \alpha_{j}\right\} ; \\
B_{l} & =B_{l-1} \cup C_{l}^{\prime \prime} .
\end{aligned}
$$

The $\leq$-based filtration of $B$ is the set $B_{\leq}=B_{m}$.

If $D$ is a set of sentences such that $D \subseteq C n(\emptyset)$ then the $\leq$-based filtration of $D$ is the empty set, i.e. $D_{\leq}=\emptyset$.

Now we can recall the definition of epistemic entrenchment-based multiple contraction. 
Definition 2.16 ([3, 14]) Let $\mathbf{K}$ be a belief set and $\leq$ be an epistemic entrenchment relation with respect to $\mathbf{K}$. The $\leq$-based multiple contraction on $\boldsymbol{K}$ is the multiple contraction function $\div \leq$ defined by:

$$
\mathbf{K} \div{ }_{\leq} B= \begin{cases}\left\{\beta \in \mathbf{K}: \forall \alpha_{j} \in B_{\leq} \alpha_{j}<\alpha_{j} \vee \beta\right\} & , \text { if } B \cap C n(\emptyset)=\emptyset \\ \mathbf{K} & \text {, if } B \cap C n(\emptyset) \neq \emptyset\end{cases}
$$

for any set of sentences $B$ and where $B \leq$ is the $\leq$-based filtration of $B$.

An operator $\div$ on $\mathbf{K}$ is an epistemic entrenchment-based multiple contraction on $\mathbf{K}$ if and only if there is an epistemic entrenchment relation $\leq$ with respect to $\mathbf{K}$, such that $\mathbf{K} \div B$ $=\mathbf{K} \div \leq B$, for any set of sentences $B$.

The following example is presented in order to clarify the above definition.

Example 2.17 Let $\mathcal{L}, \mathbf{K}, \alpha, \beta, \delta$ and $\epsilon$ be as stated in Example 2.13. Furthermore, let $\leq$ be an epistemic entrenchment relation with respect to $\mathbf{K}$ such that:

$$
\beta<\delta<\delta \vee \neg \beta==_{\leq} \epsilon=_{\leq} \epsilon \vee \neg \delta=_{\leq} \alpha=_{\leq} \alpha \vee \neg \beta<\top .
$$

Notice that it follows from Proposition 2.7 that an epistemic entrenchment relation $\leq$ in these conditions can be obtained from the system of spheres $\mathbb{S}$ presented in Example 2.13 by means of condition $(\leq-\mathbb{S})$.

Finally, let $\div \leq$ be the $\leq$-based multiple contraction, and consider the sets $A=\{\alpha, \beta\}$ and $B=\{\beta, \delta, \epsilon\}$.

In what follows we illustrate how to obtain the results of the $\leq$-based multiple contraction of $\mathbf{K}$ by each one of the sets $A$ and $B$.

We start by obtaining $\mathbf{K} \div \leq A$. For that we need to start by determining the set $A_{\leq}-$i.e. the $\leq$-based filtration of $A$.

Having in mind the process described in Definition 2.15 and the information regarding $\leq$ presented in (3) we obtain that:

- $\quad(A \backslash C n(\emptyset)) / \backsim=\left\{C_{1}, C_{2}\right\}$, with $C_{1}=\{\alpha\}$ and $C_{2}=\{\beta[--]\}$. Therefore, $m=2$.

- $A_{1}=C_{1}^{\prime \prime}=C_{1}^{\prime}=C_{1}=\{\alpha\}$

- $C_{2}^{\prime}=C_{2}=\{\beta\}$ (notice that $\alpha \vee \neg \beta \leq \alpha$ ).

$-\quad C_{2}^{\prime \prime}=C_{2}^{\prime}=\{\beta\}$.

- $A_{\leq}=A_{2}=A_{1} \cup C_{2}^{\prime \prime}=\{\alpha, \beta\}$.

Hence, according to Definition 2.16, we have that $\mathbf{K} \div \leq A=\left\{\theta \in \mathbf{K}: \forall \alpha_{j} \in A_{\leq} \alpha_{j}<\right.$ $\left.\alpha_{j} \vee \theta\right\}=\{\theta \in \mathbf{K}: \alpha<\alpha \vee \theta$ and $\beta<\beta \vee \theta\}$.

Now it is time to obtain $\mathbf{K} \div \leq B$. First of all we need to identify the set $B_{\leq}$, i.e. $\leq$-based filtration of $B$. So, proceeding as above, we obtain that:

- $(B \backslash C n(\emptyset)) / \backsim=\left\{C_{1}, C_{2}, C_{3}\right\}$, with $C_{1}=\{\epsilon\}, C_{2}=\{\delta\}$ and $C_{3}=\{\beta\}$. Therefore, $m=3$.

- $\quad B_{1}=C_{1}^{\prime \prime}=C_{1}^{\prime}=C_{1}=\{\epsilon\}$

- $C_{2}^{\prime}=C_{2}=\{\delta\}$ (notice that $\epsilon \vee \neg \delta \leq \epsilon$ ).

- $C_{2}^{\prime \prime}=C_{2}^{\prime}=\{\delta\}$.

- $B_{2}=B_{1} \cup C_{2}^{\prime \prime}=\{\epsilon, \delta\}$.

- $C_{3}^{\prime}=C_{3} \backslash\{\beta\}=\emptyset$ (notice that $\beta \notin C_{3}^{\prime}$ because $\delta \vee \neg \beta \not \leq \delta$ ).

- $C_{3}^{\prime \prime}=C_{3}^{\prime}=\emptyset$.

$-\quad B_{\mathbb{S}}=B_{3}=B_{2} \cup C_{3}^{\prime \prime}=\{\epsilon, \delta\}$. 
Thus, having in mind Definition 2.16, it follows from the above that $\mathbf{K} \div \leq B=\{\theta \in \mathbf{K}$ : $\left.\forall \alpha_{j} \in B_{\leq} \alpha_{j}<\alpha_{j} \vee \theta\right\}=\{\theta \in \mathbf{K}: \epsilon<\epsilon \vee \theta$ and $\delta<\delta \vee \theta\}$

At last, the following proposition asserts that the epistemic entrenchment-based multiple contractions are indeed a generalization of the epistemic entrenchment-based (singleton) contractions.

Proposition 2.18 ([3, 14]) Let $\boldsymbol{K}$ be a belief set and $\leq$ be an epistemic entrenchment relation with respect to $\boldsymbol{K}$. If $\div \leq$ is the $\leq$-based multiple contraction and $-\leq$ is the $\leq$-based (singleton) contraction, then:

- For any set of sentences $B$ such that $B \cap C n(\emptyset)=\emptyset$ it holds that:

$$
\boldsymbol{K} \div \leq B=\bigcap_{\alpha_{i} \in B_{\leq}} \boldsymbol{K} \div \leq\left\{\alpha_{i}\right\}=\bigcap_{\alpha_{i} \in B_{\leq}} \boldsymbol{K}-\leq \alpha_{i},
$$

where $B_{\leq}$is the $\leq$-based filtration of $B$.

- The identity $\boldsymbol{K} \div \leq\{\alpha\}=\boldsymbol{K}-_{\leq} \alpha$ is satisfied for any sentence $\alpha$.

\subsubsection{An axiomatic characterization for epistemic entrenchment-based multiple} contractions

In $[3,14]$ the following representation theorem for epistemic entrenchment-based multiple contraction was provided:

Proposition 2.19 ([3, 14]) Let $\boldsymbol{K}$ be a belief set. A multiple contraction function $\div$ on $\boldsymbol{K}$ is an epistemic entrenchment-based multiple contraction if and only if it satisfies the following postulates:

Package success If $B \cap C n(\emptyset)=\emptyset$, then $B \cap K \div B=\emptyset$.

Package inclusion $K \div B \subseteq K$.

Package relevance If $\beta \in \boldsymbol{K}$ and $\beta \notin \boldsymbol{K} \div B$, then there is a set $K^{\prime}$ such that $\boldsymbol{K} \div B \subseteq$ $K^{\prime} \subseteq K$ and $B \cap C n\left(K^{\prime}\right)=\emptyset$ but $B \cap C n\left(K^{\prime} \cup\{\beta\}\right) \neq \emptyset$.

Package uniformity If every subset $X$ of $\boldsymbol{K}$ implies some element of $B$ if and only if $X$ implies some element of $C$, then $K \div B=K \div C$.

Package conjunctive overlap $\boldsymbol{K} \div\{\alpha\} \cap \boldsymbol{K} \div\{\beta\} \subseteq \boldsymbol{K} \div\{\alpha \wedge \beta\}$.

Package conjunctive inclusion If $\alpha \notin \boldsymbol{K} \div\{\alpha \wedge \beta\}$, then $\boldsymbol{K} \div\{\alpha \wedge \beta\} \subseteq \boldsymbol{K} \div\{\alpha\}$.

Package singleton reduction For any set $B$ such that $B \cap C n(\emptyset)=\emptyset$, it holds that $\boldsymbol{K} \div B=\bigcap_{\alpha_{i} \in B_{\leq}} \boldsymbol{K} \div\left\{\alpha_{i}\right\}$, where $\leq \div$ is the epistemic entrenchment relation with respect to $\boldsymbol{K}$ defined by

$$
\alpha \leq \div \beta \text { if and only if } \alpha \notin \boldsymbol{K} \div\{\alpha \wedge \beta\} \text { or } \vdash \alpha \wedge \beta,
$$

and $B_{\leq \div}$is the $\leq_{\div}$-based filtration of $B$.

At this point it is convenient to notice that the formulation of the postulate of package singleton reduction relies on the fact that it is possible to define an epistemic entrenchment relation by means of a multiple contraction function which satisfies the remaining postulates mentioned in the above axiomatic characterization. This fact is more formally exposed in the following proposition: 
Proposition 2.20 ([3, 14]) Let $\boldsymbol{K}$ be a belief set. If $\div$ is a multiple contraction function on $\boldsymbol{K}$ that satisfies package inclusion, package success, package uniformity, package relevance, package conjunctive overlap and package conjunctive inclusion, then the binary relation $\leq$ on $\mathcal{L}$ defined from $\div$ by means of the following condition:

$$
\forall \alpha, \beta \in \mathcal{L}, \alpha \leq \beta \text { iff } \alpha \notin \boldsymbol{K} \div\{\alpha \wedge \beta\} \text { or } \vdash \alpha \wedge \beta
$$

is an epistemic entrenchment relation with respect to $\boldsymbol{K}$.

Finally, in the next proposition we recall some other multiple contraction postulates which (as a consequence of the above presented axiomatic characterization) are also satisfied by all epistemic entrenchment-based multiple contractions, and which we will need to refer to in what follows.

Proposition 2.21 ([3, 14]) Let $\boldsymbol{K}$ be a belief set and $\div$ be a multiple contraction function on $\boldsymbol{K}$. If $\div$ satisfies package inclusion, package uniformity and package relevance then it also satisfies:

Package closure $\quad K \div B=C n(K \div B)$.

Package vacuity If $B \cap K=\emptyset$, then $K \div B=K$.

Package extensionality If for every sentence $\alpha$ in $B$ there is a sentence $\beta$ in $C$ such that $\vdash \alpha \leftrightarrow \beta$, and vice versa, then $K \div B=K \div C$.

Package recovery $K \subseteq C n((K \div B) \cup B)$.

\subsubsection{Interrelation Between System of Spheres-based Multiple Contractions and Epistemic Entrenchment-based Multiple Contractions}

In what follows we recall some results of $[3,14]$ regarding the interrelation between Definitions 2.12 and 2.16, which culminate with the conclusion that the class of system of spheres-based multiple contractions coincides with the class of epistemic entrenchmentbased multiple contractions.

We start with the following proposition which asserts that if $\mathbb{S}$ and $\leq$ satisfy condition $(\leq-\mathbb{S})$ then the $\mathbb{S}$-based filtration and the $\leq$-based filtration of $B$ are identical.

Proposition 2.22 ([3, 14]) Let $\boldsymbol{K}$ be a belief set, B be a set of sentences, $\leq$ be an epistemic entrenchment relation with respect to $\boldsymbol{K}$ and $\mathbb{S}$ be a system of spheres centred on $\|\boldsymbol{K}\|$ such that condition $(\leq-\mathbb{S})$ holds. Then

$$
B_{\mathbb{S}}=B_{\leq}
$$

Combining the above proposition with Propositions 2.8, 2.14 and 2.18 we can immediately conclude the fact stated in the following proposition:

Proposition $2.23([3,14])$ Let $\boldsymbol{K}$ be a belief set, $\leq$ be an epistemic entrenchment relation with respect to $\boldsymbol{K}$ and $\mathbb{S}$ be a system of spheres centred on $\|\boldsymbol{K}\|$. Then, for any set of sentences $B$,

$$
\boldsymbol{K} \div \leq B=K \div \mathbb{S} B
$$

if and only if $\leq$ and $\mathbb{S}$ satisfy condition $(\leq-\mathbb{S})$. 
Finally, the following result which is essentially a corollary of the above proposition and Propositions 2.7 and 2.10, clarifies that the class of epistemic entrenchment-based multiple contractions coincides with the class of systems of spheres-based multiple contractions.

Proposition 2.24 ([3, 14]) Let $\boldsymbol{K}$ be a belief set. A multiple contraction function on $\boldsymbol{K}$ is an epistemic entrenchment-based multiple contraction on $\boldsymbol{K}$ if and only if it is a system of spheres-based multiple contraction on $\boldsymbol{K}$.

\section{Two new (alternative) axiomatic characterizations for the class of system of spheres-based multiple contractions}

It follows immediately from Proposition 2.24 that the multiple contraction postulates considered in Proposition 2.19 are adequate to axiomatically characterize, not only the epistemic entrenchment-based multiple contractions, but also the system of spheres-based multiple contractions. This fact is stated in the following proposition, where the postulate of package singleton reduction is formulated in terms of systems of spheres rather than in terms of epistemic entrenchment relations. We notice that the equivalence between the formulation of that postulate presented below and its original formulation (which was recalled within the statement of Proposition 2.19) can be easily shown to hold by making use of Propositions 2.9 and 2.22 .

Proposition 3.1 Let $\boldsymbol{K}$ be a belief set. A multiple contraction function $\div$ on $\boldsymbol{K}$ is a system of spheres-based multiple contraction if and only if it satisfies package success, package inclusion, package relevance, package uniformity, package conjunctive overlap, package conjunctive inclusion and

Package singleton reduction For any set $B$ such that $B \cap C n(\emptyset)=\emptyset$, it holds that $\boldsymbol{K} \div B=\bigcap_{\alpha_{i} \in B_{\mathbb{S}} \div} \boldsymbol{K} \div\left\{\alpha_{i}\right\}$, where $\mathbb{S} \div$ is a system of spheres centred on $\|\boldsymbol{K}\|$ such that

$$
\forall \alpha, \beta \in \mathcal{L} \backslash C n(\emptyset), \mathbb{S}_{\neg \alpha}^{\dot{\div}} \subseteq \mathbb{S}_{\neg \beta}^{\div} \text {if and only if } \alpha \notin \boldsymbol{K} \div\{\alpha \wedge \beta\},
$$

and $B_{\mathbb{S}} \div$ is the $\mathbb{S} \div$-based filtration of $B$.

Observing the axiomatic characterization above we immediately realize that, as it was in fact mentioned in $[3,14]$, the postulate of package singleton reduction is very technical and (at least at first sight) not at all intuitive. Furthermore, both the formulations presented for that postulate rely on some structure (in one case the epistemic entrenchment relation $\leq \div$ and in the other case the system of spheres $\mathbb{S} \div$ ) which is obtained from the multiple contraction function $\div$ under consideration in a rather intricate way.

In that regard we may notice that the formulation of that postulate in terms of a system of spheres is even more complex than the one in terms of an epistemic entrenchment relation. Indeed, while condition (4) provides an explicit definition of $\leq \div$ by means of $\div$, condition (5) does not explicitly define a system of spheres. Furthermore, taking into account Propositions 2.10 and 2.20, it follows that a way for obtaining a system of spheres $\mathbb{S} \div$ that satisfies condition (5) consists of the two following steps: 1. obtaining the epistemic entrenchment relation defined from $\div$ by means of condition $\left(C M_{\leq}\right) ; 2$. using that epistemic entrenchment relation to construct the system of spheres $\mathbb{S}^{\div}$by proceeding as described in Proposition 2.10 . 
Despite the fact that in $[3,14]$ a thorough exposition of the reasons and intuitions which motivated the formulation of the postulate of package singleton reduction has been presented, having in mind the above exposed complexity of that postulate it is obviously convenient to find another representation theorem for the system of spheres-based multiple contractions (and, consequently, also for the epistemic entrenchment-based multiple contractions), which provides us some further insight regarding the behaviour of those operations.

Thus, in this section we present two alternative axiomatic characterizations to that class of functions, which rely on some postulates which are more intuitive.

We start by introducing, in the following definition, the concept of AGM multiple contraction.

Definition 3.2 A function $\div: \mathcal{T}_{\mathcal{L}} \times 2^{\mathcal{L}} \rightarrow \mathcal{T}_{\mathcal{L}}$ that maps a theory $\mathbf{K}$ and a set of sentences $A$ to a theory $\mathbf{K} \div A$ is an $A G M$ multiple contraction (on $\mathbf{K}$ ) if, for any sentence $\alpha$, it satisfies the AGM postulates below: ${ }^{2}$

$\begin{array}{ll}(\mathbf{K} \div 1) & \mathbf{K} \div\{\alpha\} \text { is a theory (i.e. } \mathbf{K} \div\{\alpha\}=C n(\mathbf{K} \div\{\alpha\})) . \\ (\mathbf{K} \div 2) & \mathbf{K} \div\{\alpha\} \subseteq \mathbf{K} . \\ (\mathbf{K} \div 3) & \text { If }\{\alpha\} \nsubseteq \mathbf{K} \text {, then } \mathbf{K} \div\{\alpha\}=\mathbf{K} . \\ (\mathbf{K} \div 4) & \text { If } \forall \alpha, \text { then } \alpha \notin \mathbf{K} \div\{\alpha\} . \\ (\mathbf{K} \div 5) & \mathbf{K} \subseteq C n((\mathbf{K} \div\{\alpha\}) \cup\{\alpha\}) . \\ (\mathbf{K} \div 6) & \text { If } \vdash \alpha \leftrightarrow \beta, \text { then } \mathbf{K} \div\{\alpha\}=\mathbf{K} \div\{\beta\} . \\ (\mathbf{K} \div 7) & (\mathbf{K} \div\{\alpha\}) \cap(\mathbf{K} \div\{\beta\}) \subseteq \mathbf{K} \div\{\alpha \wedge \beta\} . \\ (\mathbf{K} \div 8) & \text { If } \alpha \notin \mathbf{K} \div\{\alpha \wedge \beta\} \text { then } \mathbf{K} \div\{\alpha \wedge \beta\} \subseteq \mathbf{K} \div\{\alpha\} .\end{array}$

Now, in order to be in a position to present the formulations of the remaining postulates that are necessary for the axiomatic characterization that we will present afterwards, we start by introducing the concept of primitive set of sentences (with respect to a certain multiple contraction function).

Definition 3.3 Let $\mathbf{K}$ be a belief set, $\div$ be a multiple contraction function on $\mathbf{K}$. A nonempty set $A$ of nontautological sentences is primitive (with respect to $\div$ ), if for all nonempty $A^{\prime} \subseteq A, \mathbf{K} \div A^{\prime}=\bigcap_{\alpha^{\prime} \in A^{\prime}} \mathbf{K} \div\left\{\alpha^{\prime}\right\}$.

We notice that, roughly speaking, a set of sentences $A$ is primitive if it holds that the result of the (multiple) contraction of $\mathbf{K}$ by $A$ can be obtained by intersecting the results of the singleton contractions (by means of that same multiple contraction function) of $\mathbf{K}$ by each of the sentences in $A$.

The following example clarifies the meaning of the above proposed concept.

Example 3.4 Let $\mathcal{L}, W_{0}, \ldots, W_{7}, \mathbf{K}, \mathbb{S}, \alpha, \beta, \delta$ and $\epsilon$ be as stated in Example 2.13. Furthermore, let $\div \mathbb{S}$ be the $\mathbb{S}$-based multiple contraction, and consider the sets $A=\{\alpha, \beta\}$ and $B=\{\beta, \delta, \epsilon\}$.

\footnotetext{
${ }^{2}$ In fact, to be more precise, the formulations of the postulates $(\mathbf{K} \div 1)-(\mathbf{K} \div 8)$ here presented are the result of replacing every occurrence of a single sentence by the singleton set consisting of that sentence in the formulation of the corresponding one of the AGM postulates for (singleton) contraction $(\mathbf{K}-1)-(\mathbf{K}-8)$ which we have recalled in Section 1 . We notice also that postulates $(\mathbf{K} \div 7)$ and $(\mathbf{K} \div 8)$ are the postulates that have been designated by package conjunctive overlap and package conjunctive inclusion, respectively, in Proposition 2.19.
} 
As exposed in the mentioned example, it holds that:

$$
\mathbf{K} \div \mathbb{S} A=T h\left(\left\{W_{0}, W_{1}, W_{3}\right\}\right) ; \quad \mathbf{K} \div \mathbb{S} B=T h\left(\left\{W_{0}, W_{2}, W_{3}, W_{4}\right\}\right) .
$$

On the other hand, it follows immediately from Proposition 2.14 that:

$$
\begin{aligned}
& \mathbf{K} \div \mathbb{S}\{\alpha\}=T h\left(\left\{W_{0}, W_{3}\right\}\right) ; \mathbf{K} \div \mathbb{S}\{\beta\}=T h\left(\left\{W_{0}, W_{1}\right\}\right) ; \\
& \mathbf{K} \div \mathbb{S}\{\delta\}=T h\left(\left\{W_{0}, W_{2}\right\}\right) ; \mathbf{K} \div \mathbb{S}\{\epsilon\}=T h\left(\left\{W_{0}, W_{3}, W_{4}\right\}\right) .
\end{aligned}
$$

Therefore, we can conclude that:

- The set $A=\{\alpha, \beta\}$ is primitive (with respect to $\div$ ) since, for all nonempty $A^{\prime} \subseteq A$, it holds that $\mathbf{K} \div \mathbb{S} A^{\prime}=\bigcap_{\alpha^{\prime} \in A^{\prime}} \mathbf{K} \div \mathbb{S}\left\{\alpha^{\prime}\right\}$.

- The set $B=\{\beta, \delta, \epsilon\}$ is not primitive (with respect to $\div \mathbb{S}$ ) since, for example, $\{\beta, \delta, \epsilon\} \subseteq B$ but $\mathbf{K} \div{ }_{\mathbb{S}}\{\beta, \delta, \epsilon\} \neq \mathbf{K} \div \mathbb{S}\{\beta\} \cap \mathbf{K} \div \mathbb{S}\{\delta\} \cap \mathbf{K} \div \mathbb{S}\{\epsilon\}$ (in fact, $\mathbf{K} \div \mathbb{S}\{\beta, \delta, \epsilon\}=T h\left(\left\{W_{0}, W_{2}, W_{3}, W_{4}\right\}\right)$ and $\mathbf{K} \div \mathbb{S}\{\beta\} \cap \mathbf{K} \div \mathbb{S}\{\delta\} \cap \mathbf{K} \div \mathbb{S}\{\epsilon\}=$ $\left.\operatorname{Th}\left(\left\{W_{0}, W_{1}, W_{2}, W_{3}, W_{4}\right\}\right)\right)$.

Now we introduce the following three multiple contraction postulates:

(M1) If $A$ is a primitive set and for all $\beta \in B$ there is an $\alpha \in A$ such that $(\alpha \vee \neg \beta) \in$ $\mathbf{K} \div\{\alpha\}$, then $\mathbf{K} \div(A \cup B)=\mathbf{K} \div A$.

(M2) If for all $\alpha \in A, \beta \notin \mathbf{K} \div\{\alpha \wedge \beta\}, \mathbf{K} \div\{\alpha\} \not \subset \mathbf{K} \div\{\beta\}$, and $(\alpha \vee \neg \beta) \notin \mathbf{K} \div\{\alpha\}$, then $\mathbf{K} \div(A \cup\{\beta\})=(\mathbf{K} \div A) \cap(\mathbf{K} \div\{\beta\})$.

(M3) If $A$ and $B$ are primitive, and for all $\alpha \in A$, and $\beta \in B, \alpha \in \mathbf{K} \div\{\alpha \wedge \beta\}$ and $(\alpha \vee \neg \beta) \notin \mathbf{K} \div\{\alpha\}$, then $A \cup B$ is also primitive.

Next, before making use of the above postulates to provide a new representation theorem for the system of spheres-based multiple contractions, we informally expose the intuition behind their formulations.

Thus, let $\div$ be an arbitrary multiple contraction function on a belief set $\mathbf{K}$. We start by noticing that, for any nontautological sentences $\alpha$ and $\beta$, if $(\alpha \vee \neg \beta) \in \mathbf{K} \div\{\alpha\}$ then it must be the case that $\beta \notin \mathbf{K} \div\{\alpha\}$, whereas if $(\alpha \vee \neg \beta) \notin \mathbf{K} \div\{\alpha\}$ then it may hold that $\beta \in \mathbf{K} \div\{\alpha\}$. On the other hand, since in order to remove the conjunction $\alpha \wedge \beta$ from $\mathbf{K}$ it suffices to remove one of the conjuncts, $\beta \notin \mathbf{K} \div\{\alpha \wedge \beta\}$ can be interpreted as meaning that "it is at least as easy to give up the belief $\beta$ as it is to give up $\alpha$ " and $\alpha \in \mathbf{K} \div\{\alpha \wedge \beta\}$ as meaning that "it is easier to give up the belief $\beta$ than to give up $\alpha$ ". Furthermore, the fact that $\mathbf{K} \div\{\alpha\} \not \subset \mathbf{K} \div\{\beta\}$ can be seen as meaning that $\beta$ may be contained in the set which is the result of the singleton contraction of $\mathbf{K}$ by $\alpha$.

Now, having the above interpretations in mind, it follows that the postulates (M1)-(M3) can be described as follows:

- Postulate (M1) states that, given a primitive set of sentences $A$ and a set of sentences $B$ if each of the sentences of $B$ is not contained in at least one of the sets which is a result of the singleton contraction (by means of $\div$ ) of $\mathbf{K}$ by (only) one of the sentences in $A$, then in order to (multiple) contract $\mathbf{K}$ by $A \cup B$ it is enough to (multiple) contract $\mathbf{K}$ by A;

- Postulate (M2) asserts that if $\beta$ is easier to give up than each one of the sentences in $A$ and, on the other hand, for any $\alpha \in A$ it may be the case that $\beta$ is contained in the set which is the result of the singleton contraction of $\mathbf{K}$ by $\alpha$, then the result of the multiple 
contraction of $\mathbf{K}$ by $A \cup\{\beta\}$ is obtained by intersecting the result of contracting $\mathbf{K}$ by $A$ with the result of contracting $\mathbf{K}$ by the singleton set $\{\beta\}$;

- Postulate (M3) says that, given two primitive sets $A$ and $B$, if all sentences in $B$ are easier to give up than each one of the sentences in $A$ and, furthermore, each one of the sentences in $B$ may be contained in any one of the sets which are the result of a singleton contraction of $\mathbf{K}$ by one of the elements in $A$, then $A \cup B$ is also primitive, that is, the result of the multiple contraction of $\mathbf{K}$ by $A \cup B$ coincides with the intersection of all the sets which result of contracting $\mathbf{K}$ by (a single) one of the sentences in $A \cup B$.

At this point it is also worth noticing that the postulates (M1)-(M3) are only relevant when seen as properties of a multiple contraction operator $\div$. To see this it is enough to observe that for each one of the postulates (M1)-(M3) it holds that if all the sets that occur in its statement are either empty or singleton sets and the operation $\div$ is interpreted as a singleton contraction only, then the property of $\div$ stated in that postulate is reduced to a trivial fact. Moreover, in this regard we remark also that the formulations of postulates (M1) and (M3) involve the concept of primitive set, which is only meaningful when $\div$ is a multiple contraction function, since, if $\div$ is a singleton contraction operator, then any singleton set (i.e. any of the sets to which $\div$ may be applied) is a primitive set (with respect to $\div$ ).

In a moment we shall prove that the above presented postulates $(\mathbf{K} \div 1)-(\mathbf{K} \div 8)$ and (M1)-(M3) are sound and complete with respect to the system of spheres-based construction, and to do that we will need the following auxiliary result:

Lemma 3.5 Let $\boldsymbol{K}$ be a theory, $\mathbb{S}$ a system of spheres centred on $\|\boldsymbol{K}\|$, and $\div$ the $\mathbb{S}$-based multiple contraction on $\boldsymbol{K}$. For all nonempty sets of non-tautological sentences $A$, if $A$ is primitive then the $\mathbb{S}$-based filtration of $A$ is identical to $A$, i.e. $A_{\mathbb{S}}=A$.

Proof Let $A$ be a primitive nonempty set of non-tautological sentences, and assume towards contradiction that there is a sentence $\alpha \in A$ such that $\alpha \notin A_{\mathbb{S}}$. Then there is an $\alpha^{\prime} \in A$ such that, either $f_{\mathbb{S}}\left(\neg \alpha^{\prime}\right) \subset f_{\mathbb{S}}(\neg \alpha)$, or $\mathbb{S}_{\neg \alpha} \subset \mathbb{S}_{\neg \alpha^{\prime}}$ and $f_{\mathbb{S}}\left(\neg \alpha^{\prime}\right) \subseteq\|\neg \alpha\|$. In either case, $\mathbf{K} \div\left\{\alpha, \alpha^{\prime}\right\}=\mathbf{K} \div\left\{\alpha^{\prime}\right\} \nsubseteq \mathbf{K} \div\{\alpha\}$. This of course contradicts our assumption that $A$ is primitive.

Now we are in a position to prove the two following results, which provide an axiomatic characterization for the system of spheres-based multiple contractions.

Theorem 3.6 Every system of spheres-based multiple contraction $\div$ is an AGM multiple contraction satisfying (M1)-(M3).

Proof Let $\mathbf{K}$ be a theory and $\mathbb{S}$ a system of spheres centred on $\|\mathbf{K}\|$. Let $\div$ be the $\mathbb{S}$ based multiple contraction on $\mathbf{K}$ (i.e. the multiple contraction function denoted by $\div \mathbb{S}$ in Definition 2.12). The fact that $\div$ is an AGM multiple contraction follows immediately from Propositions 3.1 and 2.21. Hence it remains only to show that $\div$ satisfies (M1)-(M3).

For (M1), let $A$ and $B$ be nonempty sets of non-tautological sentences such that for all $\beta \in B$ there is an $\alpha \in A$ such that $(\alpha \vee \neg \beta) \in \mathbf{K} \div\{\alpha\}$. Moreover assume that $A$ is primitive. Firstly we show that for every $\beta^{\prime} \in B \cap(A \cup B)_{\mathbb{S}}$, there is an $\alpha^{\prime} \in A$ such that $f_{\mathbb{S}}\left(\neg \beta^{\prime}\right)=$ $f_{\mathbb{S}}\left(\neg \alpha^{\prime}\right)$. Assume towards contradiction that this is not the case. Let $\beta$ be an element in $B \cap$ $(A \cup B)_{\mathbb{S}}$ such that for all $\alpha^{\prime} \in A, f_{\mathbb{S}}\left(\neg \beta^{\prime}\right) \neq f_{\mathbb{S}}\left(\neg \alpha^{\prime}\right)$. Moreover assume that $\beta$ is the most "distant" of such elements; i.e. for all $\beta^{\prime} \in B \cap(A \cup B)_{\mathbb{S}}$, either $\mathbb{S}_{\neg \beta^{\prime}} \subseteq \mathbb{S}_{\neg \beta}$, or there is an 
$\alpha^{\prime \prime} \in A$ such that $f_{\mathbb{S}}\left(\neg \beta^{\prime}\right)=f_{\mathbb{S}}\left(\neg \alpha^{\prime \prime}\right)$. Then since, by Lemma 3.5, $A_{\mathbb{S}}=A$, it follows that for all $\alpha^{\prime} \in A$ such that $\mathbb{S}_{\neg \beta} \subseteq \mathbb{S}_{\neg \alpha^{\prime}}, \alpha^{\prime} \in(A \cup B)_{\mathbb{S}}$. Thus, given that $\beta \in(A \cup B)_{\mathbb{S}}$, we derive that there is no $\alpha^{\prime} \in A$ such that $f_{\mathbb{S}}\left(\neg \alpha^{\prime}\right) \subset\|\neg \beta\|$ and $\mathbb{S}_{\neg \beta} \not \subset \mathbb{S}_{\neg \alpha^{\prime}}$. On the other hand according to our assumptions there is an $\alpha \in A$ such that $(\alpha \vee \neg \beta) \in \mathbf{K} \div\{\alpha\}$. Combining the above we derive that $f_{\mathbb{S}}(\neg \alpha) \subset f_{\mathbb{S}}(\neg \beta)$. Given that $\beta \in B \cap(A \cup B)_{\mathbb{S}}$ we derive that $\alpha \notin$ $(A \cup B)_{\mathbb{S}}$. Hence there is a $\beta^{\prime} \in(A \cup B)_{\mathbb{S}}$ such that either $f_{\mathbb{S}}\left(\neg \beta^{\prime}\right) \subset f_{\mathbb{S}}(\neg \alpha)$, or $\mathbb{S}_{\neg \alpha} \subset \mathbb{S}_{\neg \beta^{\prime}}$ and $f_{\mathbb{S}}\left(\neg \beta^{\prime}\right) \subset\|\neg \alpha\|$. If the former alternative holds it follows that $f_{\mathbb{S}}\left(\neg \beta^{\prime}\right) \subset f_{\mathbb{S}}(\neg \beta)$ which contradicts $\beta \in B \cap(A \cup B)_{\mathbb{S}}$. Hence the latter alternative must hold. From $\mathbb{S}_{\neg \beta}=$ $\mathbb{S}_{\neg \alpha} \subset \mathbb{S}_{\neg \beta^{\prime}}$ and $\beta^{\prime} \in(A \cup B)_{\mathbb{S}}$ we derive that there is an $\alpha^{\prime \prime} \in A$ such that $f_{\mathbb{S}}\left(\neg \beta^{\prime}\right)=$ $f_{\mathbb{S}}\left(\neg \alpha^{\prime \prime}\right)$. This, however, together with $f_{\mathbb{S}}\left(\neg \beta^{\prime}\right) \subset\|\neg \alpha\|$, contradicts the assumption that both $\alpha$ and $\alpha^{\prime \prime}$ belong to $A_{\mathbb{S}}$. Hence we can conclude that for all $\beta \in(A \cup B)_{\mathbb{S}}$, there is an $\alpha \in A$ such that $f_{\mathbb{S}}(\neg \beta)=f_{\mathbb{S}}(\neg \alpha)$. Form this, and the fact that $A=A_{\mathbb{S}}$, we can derive that $A \subseteq(A \cup B)_{\mathbb{S}}$ and, therefore, $\bigcup_{\alpha \in A_{\mathbb{S}}} f_{\mathbb{S}}(\neg \alpha) \subseteq \bigcup_{\alpha \in(A \cup B)_{\mathbb{S}}} f_{\mathbb{S}}(\neg \alpha)$. Moreover, from what we have shown above it follows that $\bigcup_{\beta \in(A \cup B)_{\mathbb{S}}} f_{\mathbb{S}}(\neg \beta) \subseteq \bigcup_{\alpha \in A_{\mathbb{S}}} f_{\mathbb{S}}(\neg \alpha)$. Hence, it holds that $\bigcup_{\beta \in(A \cup B)_{\mathbb{S}}} f_{\mathbb{S}}(\neg \beta)=\bigcup_{\alpha \in A_{\mathbb{S}}} f_{\mathbb{S}}(\neg \alpha)$ and this entails that $\mathbf{K} \div(A \cup B)=\mathbf{K} \div A$, as desired.

For (M2), let $A$ be a nonempty set of non-tautological sentences intersecting $\mathbf{K}$ and let $\beta$ be a consistent non-tautological sentence such that for all $\alpha \in A, \beta \notin \mathbf{K} \div(\alpha \wedge \beta)$, $\mathbf{K} \div\{\alpha\} \not \subset \mathbf{K} \div\{\beta\}$, and $(\alpha \vee \neg \beta) \notin \mathbf{K} \div\{\alpha\}$.

Then clearly, for all $\alpha \in A, \mathbb{S}_{\neg \beta} \subseteq \mathbb{S}_{\neg \alpha}$ and $f_{\mathbb{S}}(\neg \beta) \not \subset f_{\mathbb{S}}(\neg \alpha)$. Consequently, all elements of $A$ that belong to the $\mathbb{S}$-based filtration of $A$, also belong to the $\mathbb{S}$-based filtration of $A \cup\{\beta\}$. Furthermore, $A \cap(A \cup\{\beta\})_{\mathbb{S}}=A_{\mathbb{S}}$. On the other hand we notice that $\beta$ belongs to the $\mathbb{S}$-based filtration of $A \cup\{\beta\}$. Indeed, if it doesn't, then there must be some $\alpha \in A$ such that $f_{\mathbb{S}}(\neg \alpha) \subseteq\|\neg \beta\|$, which contradicts the assumption that for all $\alpha \in$ $A,(\alpha \vee \neg \beta) \notin \mathbf{K} \div\{\alpha\}$. Therefore we can conclude that $(A \cup\{\beta\})_{\mathbb{S}}=A_{\mathbb{S}} \cup\{\beta\}$ and it follows immediately from the definition of the $\mathbb{S}$-based multiple contraction on $\mathbf{K}$ that $\mathbf{K} \div(A \cup\{\beta\})=(\mathbf{K} \div A) \cap(\mathbf{K} \div\{\beta\})$.

Finally for (M3), assume that $A$ and $B$ are primitive and for all $\alpha \in A$, and $\beta \in B$, $\alpha \in \mathbf{K} \div\{\alpha \wedge \beta\}$ and $(\alpha \vee \neg \beta) \notin \mathbf{K} \div\{\alpha\}$. Assume now towards contradiction that $A \cup B$ is not primitive. Then there is a set $D \subseteq A \cup B$ such that $\mathbf{K} \div D \neq \bigcap_{\delta \in D} \mathbf{K} \div\{\delta\}$, and consequently, $D_{\mathbb{S}} \subset D$. Let $\psi$ be any element of $D \backslash D_{\mathbb{S}}$. Since $\psi \notin D_{\mathbb{S}}$, there is a $\theta \in D_{\mathbb{S}}$ such that either $\mathbb{S}_{\neg \psi} \subset \mathbb{S}_{\neg \theta}$ and $f_{\mathbb{S}}(\neg \theta) \subset\|\neg \psi\|$, or $f_{\mathbb{S}}(\neg \theta) \subset f_{\mathbb{S}}(\neg \psi)$. Since $A$ is primitive, it is not possible that both $\psi$ and $\theta$ belong to $A$; similarly, it is not possible for both $\psi$ and $\theta$ to belong to $B$. Consequently, either $\psi \in A$ and $\theta \in B$ or the other way around. In what follow we show that either one of those two alternatives leads to a contradiction (of our assumptions).

Case $1, \psi \in A$ and $\theta \in B$. As we have mentioned above it holds that either $\mathbb{S}_{\neg \psi} \subset \mathbb{S}_{\neg \theta}$ and $f_{\mathbb{S}}(\neg \theta) \subset\|\neg \psi\|$, or $f_{\mathbb{S}}(\neg \theta) \subset f_{\mathbb{S}}(\neg \psi)$. In either case it holds that $\mathbb{S}_{\neg \psi} \subseteq \mathbb{S}_{\neg \theta}$ and, consequently, it must be the case that $\psi \notin \mathbf{K} \div\{\psi \wedge \theta\}$, which contradicts our assumption that for all $\alpha \in A$, and $\beta \in B, \alpha \in \mathbf{K} \div\{\alpha \wedge \beta\}$.

Case 2, $\theta \in A$ and $\psi \in B$. Again it holds that either $\mathbb{S}_{\neg \psi} \subset \mathbb{S}_{\neg \theta}$ and $f_{\mathbb{S}}(\neg \theta) \subset$ $\|\neg \psi\|$, or $f_{\mathbb{S}}(\neg \theta) \subset f_{\mathbb{S}}(\neg \psi)$. In either case it holds that $f_{\mathbb{S}}(\neg \theta) \subset\|\neg \psi\|$ and, therefore $(\theta \vee \neg \psi) \in \mathbf{K} \div\{\theta\}$, which contradicts our assumption that for all $\alpha \in A$, and $\beta \in B$, $(\alpha \vee \neg \beta) \notin \mathbf{K} \div\{\alpha\}$.

Theorem 3.7 Every AGM multiple contraction $\div$ satisfying $(M 1)-(M 3)$ is a system of spheres-based multiple contraction. 
Proof Let $\mathbf{K}$ be a belief set, and $\div$ be an AGM multiple contraction (on $\mathbf{K}$ ) satisfying (M1)-(M3). For every world $W \in \mathcal{M}_{\mathcal{L}}$, define the sphere $\mathcal{V}_{W}$ as follows:

$$
\mathcal{V}_{W}=\left\{U \in \mathcal{M}_{\mathcal{L}}: U \in\|\mathbf{K} \div\{\neg((\bigwedge U) \vee(\bigwedge W))\}\|\right\}
$$

Define $\mathbb{S}$ to be the set $\mathbb{S}=\left\{\mathcal{V}_{W}: W \in \mathcal{M}_{\mathcal{L}}\right\}{ }^{3}$ From the AGM postulates of multiple contraction, it is not hard to verify that $\mathbb{S}$ is a system of spheres centred on $\|K\|$ and that, for any singleton set $D$ containing a sentence that is not a tautology,

$$
\mathbf{K} \div D=T h\left(\|\mathbf{K}\| \cup\left(\bigcup_{\delta \in D_{\mathbb{S}}} f_{\mathbb{S}}(\neg \delta)\right)\right)
$$

It suffices to show that (6) also holds when $D$ is a non-singleton set. Hence consider an arbitrary nonempty set of non-tautological sentences $D$. Let $\mathbb{N}_{\mathbb{S}}(D)$ be the set of spheres, $\mathbb{N}_{\mathbb{S}}(D)=\left\{\mathcal{V} \in \mathbb{S}:\right.$ for some $\left.\delta \in D, \mathcal{V}=\mathbb{S}_{\neg \delta}\right\}$. The proof is by induction on the number of elements $n$ of $\mathbb{N}_{\mathbb{S}}(D)$.

\section{Base Case, $\mathrm{n}=1$ :}

Assume that $\mathbb{N}_{\mathbb{S}}(D)$ has only one member. Then for all $\delta, \delta^{\prime} \in D, \mathbb{S}_{\neg \delta}=\mathbb{S}_{\neg \delta^{\prime}}$. Let $B=\{\beta \in D:$ for some $\delta \in D, \mathbf{K} \div\{\beta\} \subset \mathbf{K} \div\{\delta\}\}$, and $D^{\prime}=D \backslash B$. Let $A$ be a primitive subset of $D^{\prime}$ that is maximal w.r.t. set inclusion (i.e. no subset of $D^{\prime}$ that is a proper superset of $A$ is primitive). Firstly we show that $A=D^{\prime}$. Assume towards contradiction that there is a $\delta^{\prime} \in D^{\prime}$ such that $\delta^{\prime} \notin A$. Then, since $A \cup\left\{\delta^{\prime}\right\}$ is not primitive, there is an $A^{\prime} \subseteq A$ such that $\mathbf{K} \div\left(A^{\prime} \cup\left\{\delta^{\prime}\right\}\right) \neq \bigcap_{\psi \in A^{\prime} \cup\left\{\delta^{\prime}\right\}} \mathbf{K} \div\{\psi\}$ and, therefore, $\mathbf{K} \div\left(A^{\prime} \cup\left\{\delta^{\prime}\right\}\right) \neq\left(\mathbf{K} \div A^{\prime}\right) \cap\left(\mathbf{K} \div\left\{\delta^{\prime}\right\}\right)$. Then (M2) entails that there is an $\alpha^{\prime} \in A^{\prime}$ such that either $\delta^{\prime} \in \mathbf{K} \div\left\{\alpha^{\prime} \wedge \delta^{\prime}\right\}$ or $\mathbf{K} \div\left\{\alpha^{\prime}\right\} \subset \mathbf{K} \div\left\{\delta^{\prime}\right\}$ or $\left(\alpha^{\prime} \vee \neg \delta^{\prime}\right) \in \mathbf{K} \div\left\{\alpha^{\prime}\right\}$. The first two alternatives clearly do not hold (namely because, according to some of the above assumptions, on the one hand $\mathbb{S}_{\neg \alpha^{\prime}}=\mathbb{S}_{\neg \delta^{\prime}}$ and, therefore $\delta^{\prime} \notin \mathbf{K} \div\left\{\alpha^{\prime} \wedge \delta^{\prime}\right\}$, while, on the other hand, since $\alpha^{\prime} \in D^{\prime}$ and $\delta^{\prime} \in D^{\prime}$, it cannot be the case that $\mathbf{K} \div\left\{\alpha^{\prime}\right\} \subset$ $\left.\mathbf{K} \div\left\{\delta^{\prime}\right\}\right)$, so we conclude that $\left(\alpha^{\prime} \vee \neg \delta^{\prime}\right) \in \mathbf{K} \div\left\{\alpha^{\prime}\right\}$. This again entails that $f_{\mathbb{S}}\left(\neg \alpha^{\prime}\right) \subseteq$ $f_{\mathbb{S}}\left(\neg \delta^{\prime}\right)$ (having in mind the identity (6)). Since it is not the case that $f_{\mathbb{S}}\left(\neg \alpha^{\prime}\right) \subset f_{\mathbb{S}}\left(\neg \delta^{\prime}\right)$ (for otherwise $\delta^{\prime} \in B$ ), we derive that $f_{\mathbb{S}}\left(\neg \alpha^{\prime}\right)=f_{\mathbb{S}}\left(\neg \delta^{\prime}\right)$ and consequently, $\mathbf{K} \div\left\{\alpha^{\prime}\right\}=$ $\mathbf{K} \div\left\{\delta^{\prime}\right\}$. Given that $\mathbf{K} \div\left(A^{\prime} \cup\left\{\delta^{\prime}\right\}\right) \neq\left(\mathbf{K} \div A^{\prime}\right) \cap\left(\mathbf{K} \div\left\{\delta^{\prime}\right\}\right)$ and $A^{\prime}$ is primitive, we then derive that $\mathbf{K} \div\left(A^{\prime} \cup\left\{\delta^{\prime}\right\}\right) \neq \mathbf{K} \div A^{\prime}$. On the other hand, since $\left(\alpha^{\prime} \vee \neg \delta^{\prime}\right) \in \mathbf{K} \div\left\{\alpha^{\prime}\right\}$, (M1) entails $\mathbf{K} \div\left(A^{\prime} \cup\left\{\delta^{\prime}\right\}\right)=\mathbf{K} \div A^{\prime}$. This is a contradiction, thus we have shown that $A=D^{\prime}$ or, equivalently, that $D^{\prime}$ is primitive.

Next consider any $\beta \in B$. By construction, there is a $\delta \in D^{\prime}$ such that $\mathbf{K} \div\{\beta\} \subset \mathbf{K} \div\{\delta\}$ and, consequently, $\|\mathbf{K} \div\{\delta\}\| \subset\|\mathbf{K} \div\{\beta\}\|$. On the other hand, $\|\mathbf{K}\| \cup f_{\mathbb{S}}(\neg \delta) \subseteq\|\mathbf{K} \div\{\delta\}\|$ and $\|\mathbf{K} \div\{\beta\}\| \subseteq\|\mathbf{K}\| \cup\|\neg \beta\|$. Hence $f_{\mathbb{S}}(\neg \delta) \subseteq\|\neg \beta\|$ and, therefore $(\delta \vee \neg \beta) \in \mathbf{K} \div\{\delta\}$. Then, since $D^{\prime}$ is primitive, by (M1) we derive that $\mathbf{K} \div\left(D^{\prime} \cup B\right)=\mathbf{K} \div D^{\prime}$, and therefore $\mathbf{K} \div D=\mathbf{K} \div D^{\prime}=\bigcap_{\delta^{\prime} \in D^{\prime}} \mathbf{K} \div\left\{\delta^{\prime}\right\}=$ $T h\left(\|\mathbf{K}\| \cup\left(\bigcup_{\delta^{\prime} \in D^{\prime}} f_{\mathbb{S}}\left(\neg \delta^{\prime}\right)\right)\right)$. Then what is left to show is that $D_{\mathbb{S}}=D^{\prime}$, which however follows immediately from the assumptions of the case (or, more precisely, from the definition of $D^{\prime}$ and the assumption that $\mathbb{N}_{\mathbb{S}}(D)$ has only one member).

\footnotetext{
${ }^{3}$ If $\mathbf{K}$ is the inconsistent belief set, then $\mathbb{S}$ is defined as $\mathbb{S}=\{\emptyset\} \cup\left\{\mathcal{V}_{W}: W \in \mathcal{M}_{\mathcal{L}}\right\}$.
} 
Induction Step, $\mathrm{n}=\mathrm{k}+1$ :

Assume that $\mathbb{N}_{\mathbb{S}}(D)$ has $k+1$ members. Let $C=\left\{\delta \in D\right.$ : for all $\left.\delta^{\prime} \in D, \mathbb{S}_{\neg \delta} \subseteq \mathbb{S}_{\neg \delta^{\prime}}\right\}$. Define $A=D \backslash C$ and $B=C \cap D_{\mathbb{S}}$. Clearly $D_{\mathbb{S}}=A_{\mathbb{S}} \cup B$ and, since $B \subseteq D_{\mathbb{S}}, B_{\mathbb{S}}=$ $B$. Moreover, by the induction hypothesis, $\mathbf{K} \div A=\operatorname{Th}\left(\|\mathbf{K}\| \cup\left(\bigcup_{\alpha \in A_{\mathbb{S}}} \bar{f}_{\mathbb{S}}(\neg \alpha)\right)\right)=$ $\bigcup_{\alpha \in A_{\mathbb{S}}} \mathbf{K} \div\{\alpha\}$, and $\mathbf{K} \div B=\operatorname{Th}\left(\|\mathbf{K}\| \cup\left(\bigcup_{\beta \in B_{\mathbb{S}}} f_{\mathbb{S}}(\neg \beta)\right)\right)=\bigcup_{\beta \in B_{\mathbb{S}}} \mathbf{K} \div\{\beta\}$. Hence, again by the induction hypothesis, both $A_{\mathbb{S}}$ and $B_{\mathbb{S}}$ are primitive.

Consider now any $\alpha \in A_{\mathbb{S}}$ and $\beta \in B$. Then $\mathbb{S}_{\neg \beta} \subset \mathbb{S}_{\neg \alpha}$ and therefore $\alpha \in \mathbf{K} \div\{\alpha \wedge \beta\}$ (according to (6)). Moreover, since $\alpha, \beta \in D_{\mathbb{S}}$, we derive that $f_{\mathbb{S}}(\neg \alpha) \nsubseteq\|\neg \beta\|$ and, consequently, $(\alpha \vee \neg \beta) \notin \mathbf{K} \div\{\alpha\}$. Then by (M3) we derive that $A_{\mathbb{S}} \cup B$ is primitive and, therefore, $\mathbf{K} \div D_{\mathbb{S}}=\mathbf{K} \div\left(A_{\mathbb{S}} \cup B\right)=\bigcap_{\psi \in A_{\mathbb{S}} \cup B} \mathbf{K} \div\{\psi\}=$ $T h\left(\|\mathbf{K}\| \cup\left(\bigcup_{\psi \in A_{\mathbb{S}} \cup B} f_{\mathbb{S}}(\neg \psi)\right)\right)=T h\left(\|\mathbf{K}\| \cup\left(\bigcup_{\delta \in D_{\mathbb{S}}} f_{\mathbb{S}}(\neg \delta)\right)\right)$. So to complete the proof all we need is to show that $\mathbf{K} \div D=\mathbf{K} \div D_{\mathbb{S}}$.

Let $\delta$ be any element in $D \backslash D_{\mathbb{S}}$. Then there is a $\delta^{\prime} \in D_{\mathbb{S}}$ such that either $f_{\mathbb{S}}\left(\neg \delta^{\prime}\right) \subset$ $f_{\mathbb{S}}(\neg \delta)$, or $f_{\mathbb{S}}\left(\neg \delta^{\prime}\right) \subseteq\|\neg \delta\|$. In either case $\left(\delta^{\prime} \vee \neg \delta\right) \in \mathbf{K} \div\left\{\delta^{\prime}\right\}$ and therefore by (M1), $\mathbf{K} \div D=\mathbf{K} \div D_{\mathbb{S}}$ as desired.

An immediate consequence of the two above theorems is the following axiomatic characterization for the system of spheres-based multiple contractions (alternative to the one presented in Proposition 3.1).

Corollary 3.8 Let $\boldsymbol{K}$ be a belief set. A multiple contraction function $\div$ on $\boldsymbol{K}$ is a system of spheres-based multiple contraction if and only if it satisfies the following postulates: $(\boldsymbol{K} \div 1)$, $(\boldsymbol{K} \div 2)$, $(\boldsymbol{K} \div 3)$, $(\boldsymbol{K} \div 4),(\boldsymbol{K} \div 5),(\boldsymbol{K} \div 6),(\boldsymbol{K} \div 7),(\boldsymbol{K} \div 8),(M 1),(M 2)$ and $(M 3)$.

Furthermore, combining the above result with Propositions 2.21 and 3.1 we obtain yet the following alternative axiomatic characterization for the system of spheres-based multiple contractions (which makes use of some postulates that are more commonly used in most of the presently known axiomatic characterizations of multiple contractions).

Corollary 3.9 Let $\boldsymbol{K}$ be a belief set and $\div$ be a multiple contraction function on $\boldsymbol{K}$. Then $\div$ is a system of spheres-based multiple contraction if and only if it satisfies package inclusion, package success, package uniformity, package relevance, $(\boldsymbol{K} \div 7),(\boldsymbol{K} \div 8),(M 1),(M 2)$ and (M3).

Finally, having in mind Proposition 2.24, we notice that Corollaries 3.8 and 3.9 provide also two axiomatic characterizations for the epistemic entrenchment-based multiple contractions (both different from the one that we have recalled in Proposition 2.19. This fact is explicitly stated in the following result.

Corollary 3.10 Let $\boldsymbol{K}$ be a belief set and $\div$ be a multiple contraction function on $\boldsymbol{K}$. Then the following statements are equivalent:

1. $\div$ is an epistemic entrenchment-based multiple contraction on $\boldsymbol{K}$.

2. $\div$ satisfies $(\boldsymbol{K} \div 1),(\boldsymbol{K} \div 2),(\boldsymbol{K} \div 3),(\boldsymbol{K} \div 4),(\boldsymbol{K} \div 5),(\boldsymbol{K} \div 6),(\boldsymbol{K} \div 7),(\boldsymbol{K} \div 8),(M 1),(M 2)$ and (M3).

3 . $\div$ satisfies package inclusion, package success, package uniformity, package relevance, $(\boldsymbol{K} \div 7)$, $(\boldsymbol{K} \div 8)$, (M1), (M2) and (M3). 


\section{Conclusion and discussion}

We have presented two axiomatic characterizations of the system of spheres-based multiple contractions and, consequently, also of the epistemic entrenchment-based multiple contractions, both of them different and more natural than the one that has been presented in [3, 14].

The first one of those representation theorems appears in Corollary 3.8 and makes use of two distinct sets of postulates, namely postulates $(\mathbf{K} \div 1)-(\mathbf{K} \div 8)$ and postulates (M1)-(M3). Regarding those postulates, on the one hand we have that $(\mathbf{K} \div 1)-(\mathbf{K} \div 8)$ are straightforward adaptations of the basic and supplementary AGM postulates for (singleton) contraction to the case of multiple contraction. On the other hand, having in mind the informal meaning of each of the postulates (M1)-(M3) that we have presented right after introducing them, we notice that those are properties that we might naturally expect to be satisfied by a reasonable multiple contraction function.

The other axiomatic characterization for those two classes of multiple contraction functions was introduced in Corollary 3.9 and only differs from the above described one in the following: it uses the postulates of package inclusion, package success, package uniformity, package relevance instead of the postulates $(\mathbf{K} \div 1)-(\mathbf{K} \div 6)$. We notice that those four postulates are precisely the postulates included in the well-known axiomatic characterization of the partial meet multiple contraction that was presented in [5]. Thus, it follows from this result that the class of system of spheres-based multiple contractions (which is identical to the class of epistemic entrenchment-based multiple contractions) coincides with the class of partial meet multiple contractions that, additionally, satisfy the postulates $(\mathbf{K} \div 7),(\mathbf{K} \div 8)$, (M1), (M2) and (M3).

At this point it is worth remarking that an interesting corollary of these two axiomatic characterizations is the fact that under the assumption that a multiple contraction function $\div$ satisfies postulates $(\mathbf{K} \div 7),(\mathbf{K} \div 8)$, (M1), (M2) and (M3) it can be shown that $\div$ satisfies postulates $(\mathbf{K} \div 1)-(\mathbf{K} \div 6)$ if and only if it satisfies package inclusion, package success, package uniformity and package relevance. Next we state this fact more precisely.

Corollary 4.1 Let $\boldsymbol{K}$ be a belief set and $\div$ be a multiple contraction function on $\boldsymbol{K}$ that satisfies $(\boldsymbol{K} \div 7),(\boldsymbol{K} \div 8),(M 1),(M 2)$ and (M3). Then the following statements are equivalent:

1. $\div$ satisfies $(\boldsymbol{K} \div 1),(\boldsymbol{K} \div 2),(\boldsymbol{K} \div 3),(\boldsymbol{K} \div 4),(\boldsymbol{K} \div 5)$ and $(\boldsymbol{K} \div 6)$.

$2 . \div$ satisfies package inclusion, package success, package uniformity and package relevance.

Now, we clarify in which sense we can say that the axiomatic characterizations of the system of spheres-based multiple contractions presented in this paper are more natural than the one that has been presented in $[3,14]$ (and which we have recalled in Propositions 2.19 and 3.1). In that regard, we start by observing that the difference between the above mentioned axiomatic characterization of $[3,14]$ and the second one of the axiomatic characterizations that we have obtained in this paper is that the latter uses the postulates (M1)-(M3) instead of the postulate of package singleton reduction that had been used in the former. Thus, in support of our statement that the newly proposed axiomatic characterizations are more intuitive than the one from [3,14] we highlight the following: on the one hand postulates (M1)-(M3) consist of properties which are intuitively appropriate to be required from a plausible multiple contraction function. This remark is supported by the description of the idea that motivated the formulations of those postulates that we have presented immediately 
after introducing them. While, on the other hand the postulate of package singleton reduction is a quite technical property which, and as it was indeed already mentioned in [3, 14], has an arguably unnatural formulation.

Furthermore, while the formulations of the postulates (M1)-(M3) are independent of any other postulates, the formulation of the postulate of package singleton reduction depends on the assumption that the multiple contraction $\div$ to which such property refers to, also satisfies the postulates package inclusion, package success, package uniformity, package relevance, $(\mathbf{K} \div 7)$ and $(\mathbf{K} \div 8)$ (for the reason exposed after Proposition 2.19).

Having this in mind we notice that another relevant consequence of the axiomatic characterization that we have presented as Corollary 3.9 is the fact that it provides some further intuition and meaning to the postulate of package singleton reduction, namely because combining that corollary with Proposition 3.1 we can conclude that if a multiple contraction $\div$ satisfies package inclusion, package success, package uniformity, package relevance, $(\mathbf{K} \div 7)$ and $(\mathbf{K} \div 8)$ then it satisfies package singleton reduction if and only if it satisfies postulates (M1)-(M3). This fact is more formally stated in the following corollary:

Corollary 4.2 Let $\boldsymbol{K}$ be a belief set and $\div$ be a multiple contraction function on $\boldsymbol{K}$ that satisfies package inclusion, package success, package uniformity, package relevance, $(\boldsymbol{K} \div 7)$ and $(\boldsymbol{K} \div 8)$. Then the following statements are equivalent:

1. $\div$ satisfies (M1), (M2) and (M3).

2 . $\div$ satisfies package singleton reduction.

It is also worth to remark that it follows from Corollaries 4.1 and 4.2 that any AGM multiple contraction (cf. Definition 3.2) that satisfies the postulates (M1)-(M3) also satisfies the postulate of package singleton reduction.

Finally, to close this paper, we briefly expose the connection between this work and the study recently presented in [11].

In the mentioned reference, Hansson has studied conditions under which a partial meet multiple contraction is such that, for any set $A$ there is a set $B$ such that the result of the (multiple) contraction by $A$ can be obtained by intersecting the results of singleton contractions (by means of some partial meet singleton contraction function) by each of the sentences in $B$. More formally, Hansson investigated the possibility of a partial meet multiple contraction $\div$ on a belief set $\mathbf{K}$ being reconstructed in terms of singleton contractions in the sense that the following condition would hold:

(M-S) For each set of sentences $A$ there is a set $B$ such that

$$
\mathbf{K} \div A=\bigcap_{\beta \in B} \mathbf{K} \div^{\prime} \beta
$$

where $\mathbf{K}$ is a belief set, $\div$ is a partial meet multiple contraction, and $\div^{\prime}$ is a partial meet singleton contraction on $\mathbf{K}$.

In that paper, among other results, several conditions (regarding the sets $A$ and $B$ ) have been presented under which the above condition holds.

Now we highlight that, having in mind that the system of spheres-based singleton (respectively, multiple) contractions are partial meet singleton (resp., multiple) contractions (Cf. [8] (resp., [2, 14])), it follows that the system of spheres-based multiple contractions 
constitute a class of partial meet multiple contractions for which condition (M-S) holds. Indeed, given a belief set $\mathbf{K}$ and a system of spheres $\mathbb{S}$ centred on $\|\mathbf{K}\|$, if $\div$ is the $\mathbb{S}$-based multiple contraction on $\mathbf{K}$ then, according to Proposition 2.14, for any set of sentences $A$, if $B=A_{\mathbb{S}}$ and $\div^{\prime}$ is the $\mathbb{S}$-based (singleton) contraction on $\mathbf{K}$, then condition (7) holds.

Hence, considering the above context, we can say that in the present paper we have presented some alternative axiomatic characterizations for a class of partial meet multiple contractions which are such that each one of the results of the multiple contraction by means of that function can be reconstructed as the intersection of some (appropriately chosen) results of singleton contractions by means of an (adequate) partial meet singleton contraction.

Acknowledgments We wish to thank the three anonymous referees for their valuable comments which have led to some improvements in the paper.

\section{References}

1. Alchourrón, C., Gärdenfors, P., Makinson, D.: On the logic of theory change: partial meet contraction and revision functions. J. Symb. Log. 50, 510-530 (1985)

2. Fermé, E., Reis, M.D.L.: System of spheres-based multiple contractions. J. Philos. Log. 41, 29-52 (2012)

3. Fermé, E., Reis, M.D.L.: Epistemic entrenchment-based multiple contractions. Rev. Symb. Log. 6, 460487 (2013). doi:10.1017/S1755020313000105

4. Fuhrmann, A.: Relevant logics, modal logics and theory change. Ph.D. thesis, Australian National University, Camberra (1988)

5. Fuhrmann, A., Hansson, S.O.: A survey of multiple contraction. J. Log. Lang. Inf. 3, 39-74 (1994)

6. Gärdenfors, P.: Knowledge in flux: modeling the dynamics of epistemic states. MIT Press, Cambridge (1988)

7. Gärdenfors, P., Makinson, D.: Revisions of knowledge systems using epistemic entrenchment. In: Vardi, M.Y. (ed.) Proceedings of the second conference on theoretical aspects of reasoning about knowledge, pp. 83-95. Morgan Kaufmann, Los Altos (1988)

8. Grove, A.: Two modellings for theory change. J. Philos. Log. 17, 157-170 (1988)

9. Hansson, S.O.: New operators for theory change. Theoria 55, 114-132 (1989)

10. Hansson, S.O.: A textbook of belief dynamics. Theory change and database updating, applied logic series, vol. 11. Kluwer Academic Publishers, Dordrecht (1999)

11. Hansson, S.O.: Decomposition of multiple AGM contraction: possibility and impossibility results. Log. J. IGPL 22(4), 696-710 (2014). doi:10.1093/jigpal/jzu014

12. Niederée, R.: Multiple contraction: A further case against Gärdenfors' principle of recovery. In: Fuhrmann, A., Morreau, M. (eds.) The logic of theory change, pp. 322-334. Springer, Berlin (1991)

13. Peppas, P., Williams, M.A.: Constructive modelings for theory change. Notre Dame Journal of Formal Logic 36(1), 120-133 (1995)

14. Reis, M.D.L.: On theory multiple contraction. Ph.D. thesis, Universidade da Madeira, Funchal (2011). http://hdl.handle.net/10400.13/255

15. Reis, M.D.L.: On the interrelation between systems of spheres and epistemic entrenchment relations. Log. J. IGPL 22(1), 126-146 (2014). doi:10.1093/jigpal/jzt037 